\title{
Unsaturation in Binuclear Cyclopentadienyliron Carbonyls
}

\author{
Hongyan Wang, ${ }^{a, b}$ Yaoming Xie, ${ }^{b}$ R. Bruce King, ${ }^{b}$ and Henry F. Schaefer III \\ atomic and Molecular Physics Institute, Sichuan University \\ Chengdu 610065, P. R. China \\ and \\ bDepartment of Chemistry and Center for Computational Chemistry \\ University of Georgia, Athens, Georgia 30602, USA
}

\section{Supporting Information}

Table S1. Harmonic vibrational frequencies (in $\mathrm{cm}^{-1}$ ) and infrared intensities (in parentheses in $\mathrm{km} / \mathrm{mol}$ ) for singlet $C_{s}$ dibridged trans $-\mathrm{Cp}_{2} \mathrm{Fe}_{2}(\mathrm{CO})_{2}(\mu-\mathrm{CO})_{2}$ (Ia)

Table S2. Harmonic vibrational frequencies (in $\mathrm{cm}^{-1}$ ) and infrared intensities (in parentheses in $\mathrm{km} / \mathrm{mol}$ ) for singlet $C_{2 v}$ dibridged cis- $\mathrm{Cp}_{2} \mathrm{Fe}_{2}(\mathrm{CO})_{2}(\mu-\mathrm{CO})_{2}(\mathbf{I b})$

Table S3. Harmonic vibrational frequencies (in $\mathrm{cm}^{-1}$ ) and infrared intensities (in parentheses in $\mathrm{km} / \mathrm{mol}$ ) for singlet $C_{2 h}$ unbridged trans $-\mathrm{Cp}_{2} \mathrm{Fe}_{2}(\mathrm{CO})_{4}(\mathbf{I c})$

Table S4. Harmonic vibrational frequencies (in $\mathrm{cm}^{-1}$ ) and infrared intensities (in parentheses in $\mathrm{km} / \mathrm{mol}$ ) for triplet $C_{2 v}$ tribridged $\mathrm{Cp}_{2} \mathrm{Fe}_{2}(\mu-\mathrm{CO})_{3}(\mathbf{I I a})$

Table S5. Harmonic vibrational frequencies (in $\mathrm{cm}^{-1}$ ) and infrared intensities (in parentheses in $\mathrm{km} / \mathrm{mol})$ for singlet $C_{\mathrm{s}}$ tribridged $\mathrm{Cp}_{2} \mathrm{Fe}_{2}(\mu-\mathrm{CO})_{3}(\mathbf{I I b})$

Table S6. Harmonic vibrational frequencies (in $\mathrm{cm}^{-1}$ ) and infrared intensities (in parentheses in $\mathrm{km} / \mathrm{mol}$ ) for triplet $C_{2 v}$ singly bridged $\mathrm{Cp}_{2} \mathrm{Fe}_{2}(\mathrm{CO})_{2}(\mu-\mathrm{CO})($ IIc)

Table S7. Harmonic vibrational frequencies (in $\mathrm{cm}^{-1}$ ) and infrared intensities (in parentheses in $\mathrm{km} / \mathrm{mol}$ ) for singlet $C_{2 v}$ singly bridged $\mathrm{Cp}_{2} \mathrm{Fe}_{2}(\mathrm{CO})_{2}(\mu-\mathrm{CO})($ IId $)$

Table S8. Harmonic vibrational frequencies (in $\mathrm{cm}^{-1}$ ) and infrared intensities (in parentheses in $\mathrm{km} / \mathrm{mol}$ ) for singlet $C_{2 v}$ dibridged $\mathrm{Cp}_{2} \mathrm{Fe}_{2}(\mu-\mathrm{CO})_{2}$ (IIIa)

Table S9. Harmonic vibrational frequencies (in $\mathrm{cm}^{-1}$ ) and infrared intensities (in parentheses in $\mathrm{km} / \mathrm{mol}$ ) for triplet $C_{\mathrm{s}}$ dibridged $\mathrm{Cp}_{2} \mathrm{Fe}_{2}(\mu-\mathrm{CO})_{2}$ (IIIb)

Table S10. Harmonic vibrational frequencies (in $\mathrm{cm}^{-1}$ ) and infrared intensities (in parentheses in $\mathrm{km} / \mathrm{mol}$ ) for singlet $C_{2 h}$ trans-unbridged $\mathrm{Cp}_{2} \mathrm{Fe}_{2}(\mathrm{CO})_{2}$ (IIIc) 
Table S11. Harmonic vibrational frequencies (in $\mathrm{cm}^{-1}$ ) and infrared intensities (in parentheses in $\mathrm{km} / \mathrm{mol}$ ) for triplet $C_{i}$ trans-unbridged $\mathrm{Cp}_{2} \mathrm{Fe}_{2}(\mathrm{CO})_{2}$ (IIId)

Table S12. Harmonic vibrational frequencies (in $\mathrm{cm}^{-1}$ ) and infrared intensities (in parentheses in $\mathrm{km} / \mathrm{mol}$ ) for singlet $C_{2 v}$ cis-unbridged $\mathrm{Cp}_{2} \mathrm{Fe}_{2}(\mathrm{CO})_{2}$ (IIIe)

Table S13. Harmonic vibrational frequencies (in $\mathrm{cm}^{-1}$ ) and infrared intensities (in parentheses in $\mathrm{km} / \mathrm{mol}$ ) for triplet $C_{2 v}$ cis-unbridged $\mathrm{Cp}_{2} \mathrm{Fe}_{2}(\mathrm{CO})_{2}$ (IIIf)

Table S14. Harmonic vibrational frequencies (in $\mathrm{cm}^{-1}$ ) and infrared intensities (in parentheses in $\mathrm{km} / \mathrm{mol}$ ) for triplet $C_{s}$ monobridged $\mathrm{Cp}_{2} \mathrm{Fe}_{2}(\mathrm{CO})(\mu-\mathrm{CO})(\mathbf{I I I}$ )

Table S15. Harmonic vibrational frequencies (in $\mathrm{cm}^{-1}$ ) and infrared intensities (in parentheses in $\mathrm{km} / \mathrm{mol}$ ) for triplet $C_{1}$ bridged $\mathrm{Cp}_{2} \mathrm{Fe}_{2}(\mathrm{CO})(\mathbf{I V a})$

Table S16. Harmonic vibrational frequencies (in $\mathrm{cm}^{-1}$ ) and infrared intensities (in parentheses in $\mathrm{km} / \mathrm{mol}$ ) for singlet $C$ s bridged $\mathrm{Cp}_{2} \mathrm{Fe}_{2}(\mu-\mathrm{CO})(\mathbf{I V b})$

Table S17. Harmonic vibrational frequencies (in $\mathrm{cm}^{-1}$ ) and infrared intensities (in parentheses in $\mathrm{km} / \mathrm{mol}$ ) for singlet $C_{1}$ unbridged $\mathrm{Cp}_{2} \mathrm{Fe}_{2}(\mathrm{CO})($ IVc)

Figure 1. The $17 \mathrm{Cp}_{2} \mathrm{Fe}_{2}(\mathrm{CO})_{n}(n=4,3,2,1)$ structures discussed in this paper. 
Table S1. Harmonic vibrational frequencies (in $\mathrm{cm}^{-1}$ ) and infrared intensities (in parentheses in $\mathrm{km} / \mathrm{mol}$ ) for singlet $C_{s}$ dibridged trans $-\mathrm{Cp}_{2} \mathrm{Fe}_{2}(\mathrm{CO})_{2}(\mu-\mathrm{CO})_{2}(\mathbf{I a})$

\begin{tabular}{|c|c|c|c|c|c|}
\hline & B3LYP & BP86 & & B3LYP & BP86 \\
\hline \multirow[t]{47}{*}{$a^{\prime}$} & $58(1)$ & $59(2)$ & a" & $16(0)$ & $20(0)$ \\
\hline & $97(0)$ & $95(0)$ & & $30(0)$ & $36(0)$ \\
\hline & $106(0)$ & $104(0)$ & & $63(1)$ & $62(2)$ \\
\hline & $142(1)$ & $151(0)$ & & $95(0)$ & $93(0)$ \\
\hline & $154(0)$ & $139(1)$ & & $124(0)$ & $124(1)$ \\
\hline & $225(0)$ & $226(0)$ & & $142(0)$ & $153(0)$ \\
\hline & $236(2)$ & $233(2)$ & & $248(0)$ & $259(0)$ \\
\hline & $320(7)$ & $315(9)$ & & $309(1)$ & $320(1)$ \\
\hline & $334(0)$ & $331(0)$ & & $324(11)$ & $340(1)$ \\
\hline & $367(6)$ & $368(1)$ & & $381(1)$ & $383(0)$ \\
\hline & $407(1)$ & $402(1)$ & & $449(3)$ & $452(1)$ \\
\hline & $439(0)$ & $445(0)$ & & $512(0)$ & $511(0)$ \\
\hline & $491(2)$ & $499(6)$ & & $568(35)$ & $566(0)$ \\
\hline & $540(3)$ & $556(46)$ & & $587(1)$ & $558(12)$ \\
\hline & $548(75)$ & $568(2)$ & & $599(11)$ & $581(27)$ \\
\hline & $577(1)$ & $566(2)$ & & $617(1)$ & $606(0)$ \\
\hline & $593(15)$ & $570(3)$ & & $831(5)$ & $799(5)$ \\
\hline & $597(4)$ & $583(2)$ & & $842(2)$ & $811(2)$ \\
\hline & $604(41)$ & $607(51)$ & & $846(0)$ & $815(0)$ \\
\hline & $677(660)$ & $675(547)$ & & $851(5)$ & $819(5)$ \\
\hline & $835(76)$ & $804(65)$ & & $927(3)$ & $884(3)$ \\
\hline & $839(48)$ & $809(42)$ & & $928(0)$ & $885(0)$ \\
\hline & $845(0)$ & $814(1)$ & & $1025(10)$ & $995(10)$ \\
\hline & $850(42)$ & $820(42)$ & & $1031(6)$ & 1001(6) \\
\hline & $860(5)$ & $830(6)$ & & $1073(1)$ & $1042(1)$ \\
\hline & $872(6)$ & $841(6)$ & & $1076(1)$ & $1045(0)$ \\
\hline & $933(5)$ & $892(0)$ & & $1275(0)$ & $1225(0)$ \\
\hline & $934(0)$ & $896(4)$ & & $1277(0)$ & $1226(0)$ \\
\hline & $1015(9)$ & $985(7)$ & & $1402(0)$ & $1362(0)$ \\
\hline & $1024(6)$ & $992(5)$ & & $1402(1)$ & $1364(0)$ \\
\hline & $1074(1)$ & $1042(1)$ & & $1462(2)$ & $1413(2)$ \\
\hline & $1074(1)$ & $1044(0)$ & & $1470(3)$ & $1420(3)$ \\
\hline & $1140(1)$ & 1109(1) & & $1858(1125)$ & $1796(859)$ \\
\hline & $1140(1)$ & $1110(1)$ & & $3244(0)$ & $3170(0)$ \\
\hline & $1456(3)$ & $1404(4)$ & & $3245(0)$ & $3172(0)$ \\
\hline & $1456(3)$ & $1406(3)$ & & $3261(0)$ & $3186(0)$ \\
\hline & 1901(0) & $1820(0)$ & & $3261(0)$ & $3187(0)$ \\
\hline & $2037(1475)$ & 1946(1209) & & & \\
\hline & 2051(34) & 1961(37) & & & \\
\hline & $3241(1)$ & $3167(1)$ & & & \\
\hline & $3248(0)$ & $3174(0)$ & & & \\
\hline & $3255(0)$ & $3182(0)$ & & & \\
\hline & $3257(0)$ & $3183(0)$ & & & \\
\hline & $3269(0)$ & $3194(0)$ & & & \\
\hline & $3270(0)$ & $3195(0)$ & & & \\
\hline & $1402(3)$ & $1361(2)$ & & & \\
\hline & $1402(1)$ & $1364(2)$ & & & \\
\hline
\end{tabular}


Table S2. Harmonic vibrational frequencies (in $\mathrm{cm}^{-1}$ ) and infrared intensities (in parentheses in $\mathrm{km} / \mathrm{mol}$ ) for singlet $C_{2 v}$ dibridged cis- $\mathrm{Cp}_{2} \mathrm{Fe}_{2}(\mathrm{CO})_{2}(\mu-\mathrm{CO})_{2}(\mathbf{I b})$

\begin{tabular}{|c|c|c|c|c|c|}
\hline & B3LYP & BP86 & & B3LYP & BP86 \\
\hline \multirow[t]{25}{*}{$a_{1}$} & $70(1)$ & $70(1)$ & \multirow[t]{19}{*}{$b_{1}$} & 30i(0) & 36i(0) \\
\hline & $106(0)$ & $104(0)$ & & $98(0)$ & $96(0)$ \\
\hline & $144(0)$ & $144(0)$ & & 131(0) & $128(1)$ \\
\hline & $219(0)$ & $223(0)$ & & $315(10)$ & 314(11) \\
\hline & $332(1)$ & $329(3)$ & & $440(0)$ & $441(0)$ \\
\hline & $416(0)$ & $411(0)$ & & $512(29)$ & $511(21)$ \\
\hline & $443(3)$ & $449(2)$ & & $589(1)$ & $565(0)$ \\
\hline & 491(2) & $503(1)$ & & $615(21)$ & 607(20) \\
\hline & $563(24)$ & $569(8)$ & & $841(4)$ & $808(9)$ \\
\hline & $599(1)$ & $574(3)$ & & $844(10)$ & $810(4)$ \\
\hline & $617(48)$ & $618(44)$ & & 923(3) & $880(1)$ \\
\hline & $838(31)$ & $808(22)$ & & 1032(11) & 1001(12) \\
\hline & $852(27)$ & $820(27)$ & & 1075(3) & 1044(2) \\
\hline & $876(14)$ & $847(10)$ & & $1276(0)$ & $1226(0)$ \\
\hline & 939(3) & $906(7)$ & & 1404(2) & 1364(0) \\
\hline & 1013(13) & 982(11) & & 1470(5) & 1420(5) \\
\hline & 1074(0) & 1043(0) & & 1857(1132) & $1795(863)$ \\
\hline & 1141(2) & 1111(4) & & $3243(0)$ & $3169(0)$ \\
\hline & $1402(0)$ & $1362(0)$ & & $3258(0)$ & 3183(0) \\
\hline & $1454(1)$ & 1403(2) & \multirow[t]{24}{*}{$b_{2}$} & $97(0)$ & 0094(0) \\
\hline & 1898(4) & 1819(3) & & $155(0)$ & $0148(0)$ \\
\hline & 2079(1431) & 1991(1153) & & $227(1)$ & 0223(1) \\
\hline & $3248(0)$ & 3173(0) & & $325(3)$ & 0321(1) \\
\hline & 3261(0) & $3186(0)$ & & $372(2)$ & 0373(0) \\
\hline & 3279(2) & 3201(4) & & $529(42)$ & $0548(45)$ \\
\hline \multirow{18}{*}{$\mathrm{a}_{2}$} & $46 i(0)$ & $53 i(0)$ & & $565(14)$ & $0558(0)$ \\
\hline & $63(0)$ & $62(0)$ & & $591(2)$ & 0577(2) \\
\hline & $126(0)$ & $133(0)$ & & $684(674)$ & $0680(577)$ \\
\hline & $248(0)$ & $254(0)$ & & $811(48)$ & $0770(30)$ \\
\hline & $313(0)$ & $338(0)$ & & $845(0)$ & 0815(4) \\
\hline & $389(0)$ & $400(0)$ & & $853(48)$ & $0821(50)$ \\
\hline & $579(0)$ & $564(0)$ & & $915(1)$ & 0870(2) \\
\hline & $596(0)$ & $582(0)$ & & 1012(5) & 0981(4) \\
\hline & $840(0)$ & $807(0)$ & & 1073(1) & $1042(0)$ \\
\hline & $842(0)$ & $809(0)$ & & $1140(1)$ & 1109(1) \\
\hline & $921(0)$ & $879(0)$ & & 1401(3) & 1360(3) \\
\hline & $1026(0)$ & $993(0)$ & & $1452(3)$ & 1401(4) \\
\hline & $1068(0)$ & $1035(0)$ & & 2042(185) & 1956(199) \\
\hline & $1274(0)$ & $1223(0)$ & & $3247(0)$ & 3173(0) \\
\hline & $1402(0)$ & 1363(0) & & $3259(2)$ & $3185(2)$ \\
\hline & $1469(0)$ & $1418(0)$ & & $3272(0)$ & 3199(0) \\
\hline & $3243(0)$ & $3169(0)$ & & & \\
\hline & $3258(0)$ & 3183(0) & & & \\
\hline
\end{tabular}


Table S3. Harmonic vibrational frequencies (in $\mathrm{cm}^{-1}$ ) and infrared intensities (in parentheses in $\mathrm{km} / \mathrm{mol}$ ) for singlet $C_{2 h}$ unbridged trans- $\mathrm{Cp}_{2} \mathrm{Fe}_{2}(\mathrm{CO})_{4}(\mathbf{I c})$

\begin{tabular}{|c|c|c|c|c|c|}
\hline & B3LYP & BP86 & & B3LYP & BP86 \\
\hline \multirow[t]{23}{*}{$a_{g}$} & $82(0)$ & $77(0)$ & $b_{g}$ & $53(0)$ & $49(0)$ \\
\hline & 111(0) & 107(0) & & $62(0)$ & $55(0)$ \\
\hline & $119(0)$ & $119(0)$ & & $132(0)$ & $129(0)$ \\
\hline & $178(0)$ & $175(0)$ & & $362(0)$ & $364(0)$ \\
\hline & $327(0)$ & $327(0)$ & & $417(0)$ & 411(0) \\
\hline & $370(0)$ & $378(0)$ & & $498(0)$ & $494(0)$ \\
\hline & $511(0)$ & $525(0)$ & & $576(0)$ & $569(0)$ \\
\hline & $580(0)$ & $570(0)$ & & $595(0)$ & $596(0)$ \\
\hline & $601(0)$ & $581(0)$ & & $844(0)$ & $813(0)$ \\
\hline & 644(0) & $645(0)$ & & $856(0)$ & $826(0)$ \\
\hline & $842(0)$ & $811(0)$ & & $921(0)$ & $877(0)$ \\
\hline & $847(0)$ & $817(0)$ & & $1013(0)$ & $981(0)$ \\
\hline & $857(0)$ & $824(0)$ & & $1073(0)$ & $1041(0)$ \\
\hline & $936(0)$ & 893(0) & & $1273(0)$ & $1222(0)$ \\
\hline & $1029(0)$ & $997(0)$ & & 1399(0) & $1360(0)$ \\
\hline & $1072(0)$ & $1041(0)$ & & $1452(0)$ & $1400(0)$ \\
\hline & $1138(0)$ & $1106(0)$ & & 1999(0) & $1918(0)$ \\
\hline & $1404(0)$ & $1363(0)$ & & $3242(0)$ & $3168(0)$ \\
\hline & $1466(0)$ & $1413(0)$ & & $3257(0)$ & $3183(0)$ \\
\hline & $2057(0)$ & $1973(0)$ & $\mathrm{b}_{\mathrm{u}}$ & 77(1) & $76(1)$ \\
\hline & $3248(0)$ & $3174(0)$ & & $117(0)$ & $115(0)$ \\
\hline & $3259(0)$ & $3184(0)$ & & $142(5)$ & $141(5)$ \\
\hline & $3271(0)$ & $3196(0)$ & & $326(1)$ & $327(6)$ \\
\hline \multirow[t]{20}{*}{$\mathrm{a}_{\mathrm{u}}$} & $36(1)$ & 37(1) & & $360(3)$ & $369(2)$ \\
\hline & $50(0)$ & $49(0)$ & & $501(83)$ & $522(54)$ \\
\hline & $91(0)$ & $88(0)$ & & $571(247)$ & $565(200)$ \\
\hline & $122(0)$ & $119(1)$ & & $599(21)$ & $576(42)$ \\
\hline & $361(5)$ & $362(6)$ & & $47(79)$ & $647(70)$ \\
\hline & $460(1)$ & $456(1)$ & & $842(52)$ & $811(30)$ \\
\hline & $506(9)$ & 497(12) & & $846(101)$ & $816(97)$ \\
\hline & $576(70)$ & $566(4)$ & & $854(18)$ & $822(16)$ \\
\hline & 594(4) & $597(45)$ & & $934(4)$ & 891(2) \\
\hline & $844(0)$ & $812(0)$ & & $1028(8)$ & $997(10)$ \\
\hline & $855(11)$ & $825(13)$ & & $1072(2)$ & $1040(2)$ \\
\hline & $921(0)$ & $877(0)$ & & $1138(3)$ & $1106(4)$ \\
\hline & $1012(17)$ & $980(15)$ & & $1404(2)$ & $1363(0)$ \\
\hline & $1072(1)$ & 1039(2) & & $1465(8)$ & 1413(9) \\
\hline & $1273(0)$ & $1222(0)$ & & $2013(1275)$ & 1947(927) \\
\hline & $1396(4)$ & $1356(4)$ & & $3248(0)$ & $3174(0)$ \\
\hline & 1452(3) & 1400(4) & & $3259(3)$ & $3184(4)$ \\
\hline & $2016(1499)$ & 1931(1240) & & $3271(0)$ & $3196(0)$ \\
\hline & $3242(1)$ & $3168(1)$ & & & \\
\hline & $3257(0)$ & $3183(0)$ & & & \\
\hline
\end{tabular}


Table S4. Harmonic vibrational frequencies (in $\mathrm{cm}^{-1}$ ) and infrared intensities (in parentheses in $\mathrm{km} / \mathrm{mol}$ ) for triplet $C_{2 v}$ tribridged $\mathrm{Cp}_{2} \mathrm{Fe}_{2}(\mu-\mathrm{CO})_{3}$ (IIa)

\begin{tabular}{|c|c|c|c|c|c|}
\hline & B3LYP & BP86 & & B3LYP & BP86 \\
\hline \multirow[t]{23}{*}{$a_{1}$} & $81(2)$ & $78(2)$ & \multirow[t]{19}{*}{$b_{1}$} & $23 i(0)$ & $24 \mathrm{i}(0)$ \\
\hline & $110(0)$ & $108(0)$ & & $78(2)$ & $75(2)$ \\
\hline & $212(0)$ & $215(0)$ & & $115(0)$ & $113(0)$ \\
\hline & $318(9)$ & $323(9)$ & & $306(7)$ & $306(10)$ \\
\hline & $424(0)$ & $428(0)$ & & $415(0)$ & $406(0)$ \\
\hline & 493(11) & 497(6) & & 491(10) & $493(5)$ \\
\hline & $507(0)$ & $519(0)$ & & $569(24)$ & $556(15)$ \\
\hline & $575(23)$ & $562(12)$ & & $600(1)$ & $576(3)$ \\
\hline & $601(3)$ & $580(7)$ & & $844(5)$ & $813(5)$ \\
\hline & $827(0)$ & 796(0) & & $847(2)$ & $817(3)$ \\
\hline & $832(4)$ & $800(4)$ & & 915(2) & 873(3) \\
\hline & $846(0)$ & $816(0)$ & & 1023(19) & 993(17) \\
\hline & $917(1)$ & $876(1)$ & & 1073(1) & 1043(1) \\
\hline & $1020(22)$ & 991(21) & & 1274(0) & 1224(0) \\
\hline & 1073(1) & $1043(1)$ & & 1397(0) & $1360(0)$ \\
\hline & $1138(0)$ & $1110(0)$ & & 1461(5) & 1410(7) \\
\hline & $1398(0)$ & $1362(0)$ & & 1904(982) & 1819(779) \\
\hline & 1455(3) & 1406(4) & & $3241(0)$ & $3167(0)$ \\
\hline & 1905(1009) & $1820(800)$ & & $3254(0)$ & $3179(0)$ \\
\hline & 1951(0) & $1862(0)$ & \multirow[t]{20}{*}{$b_{2}$} & $96(0)$ & $128(0)$ \\
\hline & $3241(0)$ & $3167(0)$ & & $181(0)$ & 203(0) \\
\hline & $3253(0)$ & $3179(0)$ & & 201(1) & $225(0)$ \\
\hline & $3265(0)$ & $3190(0)$ & & $304(3)$ & $323(1)$ \\
\hline \multirow[t]{16}{*}{$\mathrm{a}_{2}$} & $25 i(0)$ & $25 \mathrm{i}(0)$ & & $323(88)$ & $341(20)$ \\
\hline & $105(0)$ & $137(0)$ & & $441(0)$ & $447(0)$ \\
\hline & $182(0)$ & $218(0)$ & & $555(239)$ & $558(22)$ \\
\hline & $309(0)$ & $325(0)$ & & $581(15)$ & $592(232)$ \\
\hline & $429(0)$ & $435(0)$ & & $825(153)$ & $795(111)$ \\
\hline & $579(0)$ & $559(0)$ & & $830(8)$ & 798(9) \\
\hline & $837(0)$ & $809(0)$ & & $837(11)$ & $810(9)$ \\
\hline & $843(0)$ & $814(0)$ & & $915(2)$ & $875(4)$ \\
\hline & $913(0)$ & $872(0)$ & & $1020(0)$ & $991(0)$ \\
\hline & 1023(0) & $993(0)$ & & 1069(0) & 1040(0) \\
\hline & 1070(0) & 1041(0) & & 1137(9) & 1109(7) \\
\hline & $1274(0)$ & $1224(0)$ & & 1391(0) & $1356(1)$ \\
\hline & $1390(0)$ & $1355(0)$ & & $1455(0)$ & $1406(0)$ \\
\hline & 1461(0) & $1410(0)$ & & $3241(0)$ & $3166(0)$ \\
\hline & $3241(0)$ & $3166(0)$ & & $3253(0)$ & $3179(0)$ \\
\hline & $3254(0)$ & $3179(0)$ & & $3265(0)$ & $3190(1)$ \\
\hline
\end{tabular}


Table S5. Harmonic vibrational frequencies (in $\mathrm{cm}^{-1}$ ) and infrared intensities (in parentheses in $\mathrm{km} / \mathrm{mol}$ ) for singlet $C_{\mathrm{s}}$ dibridged $\mathrm{Cp}_{2} \mathrm{Fe}_{2}(\mu-\mathrm{CO})_{2}(\mathrm{CO})(\mathbf{I I b})$

\begin{tabular}{|c|c|c|c|c|c|}
\hline & B3LYP & BP86 & & B3LYP & BP86 \\
\hline \multirow[t]{43}{*}{$\mathrm{A}^{\prime}$} & $27(1)$ & $24(2)$ & A" & 20i(0) & 31i(0) \\
\hline & $88(2)$ & $90(1)$ & & $21(0)$ & $4(0)$ \\
\hline & $105(0)$ & $118(0)$ & & $74(0)$ & $66(1)$ \\
\hline & $150(0)$ & $158(0)$ & & 117(1) & $116(1)$ \\
\hline & $200(0)$ & $203(0)$ & & $151(0)$ & $153(0)$ \\
\hline & $234(3)$ & $232(3)$ & & $256(0)$ & $256(0)$ \\
\hline & $342(3)$ & $344(3)$ & & $316(17)$ & $315(17)$ \\
\hline & $364(3)$ & $363(0)$ & & $334(0)$ & $348(4)$ \\
\hline & $384(2)$ & $397(1)$ & & $412(3)$ & $410(1)$ \\
\hline & $418(3)$ & 418(15) & & $449(0)$ & $468(4)$ \\
\hline & $453(4)$ & $457(1)$ & & $517(10)$ & $512(1)$ \\
\hline & $508(2)$ & $527(12)$ & & $564(21)$ & $540(10)$ \\
\hline & $545(37)$ & $530(12)$ & & $586(0)$ & $562(4)$ \\
\hline & $590(0)$ & $562(2)$ & & $594(2)$ & $566(9)$ \\
\hline & $595(1)$ & $571(1)$ & & $831(2)$ & 790(1) \\
\hline & 605(35) & 583(34) & & $841(0)$ & $806(7)$ \\
\hline & $696(550)$ & $674(352)$ & & 843(9) & $810(1)$ \\
\hline & $829(29)$ & $798(6)$ & & $843(2)$ & $814(4)$ \\
\hline & $834(36)$ & $803(30)$ & & $912(3)$ & $866(3)$ \\
\hline & $840(0)$ & $808(6)$ & & 924(3) & $882(2)$ \\
\hline & $848(46)$ & 819(44) & & $1025(7)$ & $993(8)$ \\
\hline & $852(6)$ & $823(12)$ & & 1032(6) & 1004(7) \\
\hline & $860(9)$ & $835(10)$ & & 1072(1) & 1041(0) \\
\hline & $916(1)$ & 872(5) & & 1076(1) & $1045(0)$ \\
\hline & $927(0)$ & $885(1)$ & & $1273(0)$ & 1223(0) \\
\hline & 1011(7) & 981(8) & & 1277(0) & $1228(0)$ \\
\hline & 1013(16) & 982(11) & & 1396(3) & 1352(0) \\
\hline & 1070(1) & 1041(1) & & 1399(0) & 1359(0) \\
\hline & 1074(1) & $1045(0)$ & & 1464(1) & 1414(1) \\
\hline & $1137(6)$ & $1105(8)$ & & 1474(3) & 1426(3) \\
\hline & 1139(1) & 1108(1) & & 1842(1158) & 1781(918) \\
\hline & $1400(3)$ & 1359(2) & & $3235(0)$ & $3161(0)$ \\
\hline & $1402(2)$ & 1361(1) & & $3244(0)$ & $3169(0)$ \\
\hline & 1449(1) & 1396(2) & & $3255(0)$ & 3179(0) \\
\hline & 1452(3) & 1401(5) & & 3263(0) & $3187(0)$ \\
\hline & 1880(29) & $1807(77)$ & & & \\
\hline & $2047(832)$ & $1949(550)$ & & & \\
\hline & $3239(0)$ & $3162(0)$ & & & \\
\hline & $3246(0)$ & $3169(0)$ & & & \\
\hline & $3247(0)$ & 3171(1) & & & \\
\hline & $3255(0)$ & $3179(0)$ & & & \\
\hline & $3263(0)$ & $3186(0)$ & & & \\
\hline & $3270(0)$ & 3194(0) & & & \\
\hline
\end{tabular}


Table S6. Harmonic vibrational frequencies (in $\mathrm{cm}^{-1}$ ) and infrared intensities (in parentheses in $\mathrm{km} / \mathrm{mol}$ ) for triplet $C_{2 v}$ singly bridged $\mathrm{Cp}_{2} \mathrm{Fe}_{2}(\mathrm{CO})_{2}(\mu-\mathrm{CO})($ IIc)

\begin{tabular}{|c|c|c|c|c|c|}
\hline & B3LYP & BP86 & & B3LYP & BP86 \\
\hline \multirow[t]{23}{*}{$a_{1}$} & $116(1)$ & $135(2)$ & \multirow[t]{17}{*}{$\mathrm{b}_{1}$} & $602 \mathrm{i}$ & $49 i$ \\
\hline & $125(0)$ & $145(0)$ & & $35 \mathrm{i}$ & $36(2)$ \\
\hline & $178(0)$ & $220(0)$ & & $55(0)$ & $122(0)$ \\
\hline & $313(5)$ & $314(15)$ & & $168(2)$ & $290(4)$ \\
\hline & $356(0)$ & $378(0)$ & & $320(5)$ & $404(1)$ \\
\hline & $378(0)$ & $410(0)$ & & $378(7)$ & $485(20)$ \\
\hline & $505(59)$ & $506(22)$ & & $572(7)$ & $560(1)$ \\
\hline & $569(13)$ & $568(0)$ & & $788(10)$ & $763(1)$ \\
\hline & $596(0)$ & $589(8)$ & & $833(5)$ & $806(0)$ \\
\hline & $815(25)$ & $785(22)$ & & $917(3)$ & $874(1)$ \\
\hline & $841(1)$ & 813(1) & & $1032(65)$ & 1003(35) \\
\hline & $878(13)$ & $844(15)$ & & $1068(0)$ & $1040(0)$ \\
\hline & $941(0)$ & $904(0)$ & & $1272(0)$ & $1222(0)$ \\
\hline & $1003(12)$ & 973(9) & & $1390(15)$ & $1353(1)$ \\
\hline & $1069(0)$ & $1037(2)$ & & 1481(2) & $1431(2)$ \\
\hline & $1137(1)$ & $1106(0)$ & & $3244(8)$ & $3175(0)$ \\
\hline & $1404(0)$ & $1356(2)$ & & $3258(8)$ & $3187(0)$ \\
\hline & 1438(3) & 1394(4) & \multirow[t]{22}{*}{$b_{2}$} & $86(2)$ & $61(2)$ \\
\hline & $1862(565)$ & $1735(433)$ & & $168(5)$ & $169(16)$ \\
\hline & $2055(1349)$ & $1954(946)$ & & $282(50)$ & 251(76) \\
\hline & $3237(1)$ & $3168(0)$ & & $303(36)$ & $300(7)$ \\
\hline & $3253(0)$ & $3182(0)$ & & $354(3)$ & $316(0)$ \\
\hline & $3268(0)$ & 3197(0) & & $400(429)$ & $466(69)$ \\
\hline \multirow[t]{16}{*}{$\mathrm{a}_{2}$} & 786i & 838i & & 498(13) & $503(8)$ \\
\hline & $56 \mathbf{i}$ & $68 i$ & & $585(228)$ & $567(0)$ \\
\hline & $45(0)$ & $42(0)$ & & $597(88)$ & $610(10)$ \\
\hline & $199(0)$ & $219(0)$ & & $814(122)$ & $780(13)$ \\
\hline & $346(0)$ & $337(0)$ & & $840(4)$ & $811(32)$ \\
\hline & $572(0)$ & $550(0)$ & & $877(2)$ & $841(25)$ \\
\hline & $774(0)$ & $741(0)$ & & $941(6)$ & $902(43)$ \\
\hline & $834(0)$ & $804(0)$ & & 1003(5) & $972(13)$ \\
\hline & $917(0)$ & $874(0)$ & & $1068(1)$ & $1036(1)$ \\
\hline & $1029(0)$ & $999(0)$ & & $1137(2)$ & 1103(6) \\
\hline & $1068(0)$ & $1039(0)$ & & $1404(14)$ & $1350(60)$ \\
\hline & $1272(0)$ & $1222(0)$ & & $1438(6)$ & $1394(13)$ \\
\hline & $1388(0)$ & 1351(0) & & 1989(85) & $1892(324)$ \\
\hline & $1481(0)$ & 1431(0) & & $3237(0)$ & $3168(5)$ \\
\hline & $3244(0)$ & $3173(0)$ & & $3253(0)$ & $3182(1)$ \\
\hline & $3257(0)$ & $3186(0)$ & & $3268(0)$ & $3197(1)$ \\
\hline
\end{tabular}


Table S7. Harmonic vibrational frequencies (in $\mathrm{cm}^{-1}$ ) and infrared intensities (in parentheses in $\mathrm{km} / \mathrm{mol}$ ) for singlet $C_{2 v}$ singly bridged $\mathrm{Cp}_{2} \mathrm{Fe}_{2}(\mathrm{CO})_{2}(\mu-\mathrm{CO})($ IId)

\begin{tabular}{|c|c|c|c|c|c|}
\hline & B3LYP & BP86 & & B3LYP & BP86 \\
\hline \multirow{23}{*}{$a_{1}$} & 141(0) & 146(1) & \multirow{17}{*}{$b_{1}$} & $32 \mathbf{i}$ & $29 \mathbf{i}$ \\
\hline & $149(1)$ & 151(1) & & $48(0)$ & $48(0)$ \\
\hline & $226(0)$ & $234(0)$ & & $135(0)$ & $132(0)$ \\
\hline & $337(16)$ & $345(21)$ & & $357(8)$ & $361(9)$ \\
\hline & $394(0)$ & $404(0)$ & & $418(3)$ & $409(3)$ \\
\hline & $416(0)$ & $423(1)$ & & $549(34)$ & $528(22)$ \\
\hline & $518(44)$ & $518(27)$ & & $590(1)$ & $560(0)$ \\
\hline & $591(4)$ & $569(1)$ & & $828(0)$ & $787(0)$ \\
\hline & $597(1)$ & $585(9)$ & & $843(0)$ & $811(0)$ \\
\hline & $835(10)$ & 797(6) & & $930(2)$ & $881(3)$ \\
\hline & $848(11)$ & $817(9)$ & & 1031(29) & $1000(28)$ \\
\hline & $885(18)$ & $851(16)$ & & $1073(0)$ & 1041(0) \\
\hline & $951(0)$ & $913(0)$ & & $1274(0)$ & $1222(0)$ \\
\hline & 1015(9) & $979(9)$ & & 1404(1) & $1363(0)$ \\
\hline & 1074(1) & $1040(1)$ & & 1471(3) & $1420(4)$ \\
\hline & 1141(0) & 1108(1) & & 3252(0) & $3174(0)$ \\
\hline & $1407(0)$ & 1370(0) & & 3264(0) & $3186(0)$ \\
\hline & $1446(4)$ & 1389(4) & \multirow[t]{22}{*}{$b_{2}$} & $94(4)$ & $89(3)$ \\
\hline & $1796(540)$ & $1730(438)$ & & $195(0)$ & 199(0) \\
\hline & 2042(1176) & 1949(954) & & $316(7)$ & $314(5)$ \\
\hline & $3246(0)$ & $3169(0)$ & & $335(2)$ & $342(2)$ \\
\hline & $3260(0)$ & 3182(0) & & $377(0)$ & $388(1)$ \\
\hline & $3275(1)$ & 3195(0) & & 463(43) & 480(91) \\
\hline \multirow[t]{16}{*}{$\mathrm{a}_{2}$} & $67 i$ & $67 i$ & & 502(23) & $521(0)$ \\
\hline & $46(0)$ & $49(0)$ & & $587(3)$ & $571(4)$ \\
\hline & $159(0)$ & $159(0)$ & & $613(45)$ & 632(99) \\
\hline & $362(0)$ & $370(0)$ & & 834(23) & $796(3)$ \\
\hline & $438(0)$ & $442(0)$ & & $847(63)$ & $815(59)$ \\
\hline & $590(0)$ & $562(0)$ & & $886(0)$ & $852(0)$ \\
\hline & $829(0)$ & $789(0)$ & & 951(18) & 913(22) \\
\hline & $844(0)$ & $813(0)$ & & $1015(1)$ & $979(2)$ \\
\hline & $930(0)$ & $881(0)$ & & 1074(2) & 1039(5) \\
\hline & 1031(0) & $1000(0)$ & & 1141(9) & 1107(11) \\
\hline & 1073(0) & 1041(0) & & $1407(0)$ & $1369(2)$ \\
\hline & $1274(0)$ & 1222(0) & & $1446(2)$ & 1389(0) \\
\hline & $1404(0)$ & 1362(0) & & 1975(363) & $1890(232)$ \\
\hline & 1471(0) & $1420(0)$ & & $3246(0)$ & $3169(1)$ \\
\hline & $3252(0)$ & $3174(0)$ & & $3260(0)$ & 3182(0) \\
\hline & $3264(0)$ & $3186(0)$ & & $3275(0)$ & $3195(0)$ \\
\hline
\end{tabular}


Table S8. Harmonic vibrational frequencies (in $\mathrm{cm}^{-1}$ ) and infrared intensities (in parentheses in $\mathrm{km} / \mathrm{mol}$ ) for singlet $C_{2 v}$ dibridged $\mathrm{Cp}_{2} \mathrm{Fe}_{2}(\mu-\mathrm{CO})_{2}$ (IIIa)

\begin{tabular}{|c|c|c|c|c|c|}
\hline & B3LYP & BP86 & & B3LYP & BP86 \\
\hline \multirow[t]{21}{*}{$a_{1}$} & $64(2)$ & $64(2)$ & \multirow[t]{17}{*}{$\mathrm{b}_{1}$} & $41(0)$ & $40(1)$ \\
\hline & $106(0)$ & $98(0)$ & & $111(0)$ & $105(0)$ \\
\hline & $214(0)$ & $217(0)$ & & $356(10)$ & $354(11)$ \\
\hline & $368(10)$ & $366(11)$ & & $448(0)$ & $429(0)$ \\
\hline & $432(1)$ & $433(0)$ & & $540(30)$ & $537(13)$ \\
\hline & $502(0)$ & $495(0)$ & & $588(1)$ & $558(4)$ \\
\hline & 581(16) & $565(0)$ & & $842(0)$ & $809(0)$ \\
\hline & $597(2)$ & 584(13) & & $863(8)$ & $828(7)$ \\
\hline & $825(0)$ & 786(0) & & $917(0)$ & $870(0)$ \\
\hline & $832(1)$ & $796(0)$ & & 1016(17) & 980(17) \\
\hline & $845(0)$ & $813(0)$ & & 1071(0) & $1039(0)$ \\
\hline & $917(0)$ & $865(1)$ & & $1274(0)$ & $1223(0)$ \\
\hline & $1027(27)$ & $997(25)$ & & 1396(0) & 1353(0) \\
\hline & 1072(0) & 1041(0) & & $1446(5)$ & $1390(6)$ \\
\hline & 1137(0) & $1105(0)$ & & 1918(1207) & 1828(899) \\
\hline & 1403(1) & $1365(0)$ & & $3240(0)$ & 3164(0) \\
\hline & 1465(3) & 1418(3) & & $3251(0)$ & $3175(0)$ \\
\hline & 1939(202) & 1851(195) & \multirow[t]{20}{*}{$b_{2}$} & $160(0)$ & $151(0)$ \\
\hline & $3240(0)$ & 3163(0) & & $195(0)$ & 211(1) \\
\hline & $3258(0)$ & 3180(1) & & $356(3)$ & $358(4)$ \\
\hline & $3266(0)$ & $3189(0)$ & & $388(10)$ & $403(7)$ \\
\hline \multirow{16}{*}{$a_{2}$} & $30(0)$ & $29(0)$ & & $453(36)$ & $499(52)$ \\
\hline & $142(0)$ & $132(0)$ & & $595(10)$ & $567(17)$ \\
\hline & 202(0) & $219(0)$ & & $827(3)$ & 787(0) \\
\hline & $338(0)$ & $351(0)$ & & $828(88)$ & 792(74) \\
\hline & $466(0)$ & $464(0)$ & & $842(15)$ & $807(8)$ \\
\hline & $585(0)$ & $553(0)$ & & $916(3)$ & $863(3)$ \\
\hline & $841(0)$ & $806(0)$ & & $1028(0)$ & $997(0)$ \\
\hline & $863(0)$ & $827(0)$ & & 1072(1) & 1040(1) \\
\hline & $916(0)$ & $868(0)$ & & 1137(17) & $1104(19)$ \\
\hline & $1015(0)$ & $980(0)$ & & 1403(0) & $1366(0)$ \\
\hline & 1069(0) & $1035(0)$ & & $1465(0)$ & 1417(1) \\
\hline & $1274(0)$ & 1222(0) & & $3240(0)$ & 3163(0) \\
\hline & 1392(0) & $1347(0)$ & & $3258(0)$ & $3181(0)$ \\
\hline & $1446(0)$ & 1390(0) & & $3266(0)$ & $3189(0)$ \\
\hline & $3240(0)$ & $3164(0)$ & & & \\
\hline & 3251(0) & $3175(0)$ & & & \\
\hline
\end{tabular}


Table S9. Harmonic vibrational frequencies (in $\mathrm{cm}^{-1}$ ) and infrared intensities (in parentheses in $\mathrm{km} / \mathrm{mol}$ ) for triplet $C_{\mathrm{s}}$ dibridged $\mathrm{Cp}_{2} \mathrm{Fe}_{2}(\mu-\mathrm{CO})_{2}($ IIIb)

\begin{tabular}{|c|c|c|c|c|c|}
\hline & B3LYP & BP86 & & B3LYP & BP86 \\
\hline \multirow[t]{39}{*}{$a^{\prime}$} & $40(1)$ & $43(1)$ & $a^{\prime \prime}$ & $91 i$ & $22 \mathrm{i}$ \\
\hline & $86(2)$ & $86(0)$ & & $35 i$ & $25(0)$ \\
\hline & $134(0)$ & 102(1) & & $28(0)$ & 101(1) \\
\hline & $161(7)$ & 197(2) & & $97(1)$ & $130(0)$ \\
\hline & $196(0)$ & 204(1) & & $158(2)$ & $247(0)$ \\
\hline & $270(2)$ & $282(2)$ & & $234(4)$ & $304(5)$ \\
\hline & $320(18)$ & $339(0)$ & & $326(0)$ & $324(12)$ \\
\hline & $346(20)$ & $347(7)$ & & $399(2)$ & 413(0) \\
\hline & 380(1) & $411(0)$ & & $420(0)$ & $448(0)$ \\
\hline & $427(65)$ & 493(1) & & $520(26)$ & $533(13)$ \\
\hline & $488(70)$ & 513(29) & & $587(0)$ & $552(2)$ \\
\hline & $536(34)$ & $559(3)$ & & $595(0)$ & $562(0)$ \\
\hline & 593(1) & $563(8)$ & & 799(2) & $796(5)$ \\
\hline & $596(4)$ & $569(16)$ & & $839(0)$ & $799(2)$ \\
\hline & $798(85)$ & $785(51)$ & & $840(0)$ & $800(0)$ \\
\hline & $804(45)$ & $790(29)$ & & $854(3)$ & $811(0)$ \\
\hline & $826(5)$ & 789(1) & & $890(0)$ & $859(0)$ \\
\hline & $831(52)$ & 798(4) & & $919(0)$ & $863(0)$ \\
\hline & $843(1)$ & $810(24)$ & & 1011(9) & $990(5)$ \\
\hline & $846(1)$ & $816(0)$ & & 1019(13) & $990(15)$ \\
\hline & $891(0)$ & $858(5)$ & & $1066(0)$ & $1036(0)$ \\
\hline & $911(2)$ & $863(0)$ & & $1068(0)$ & 1039(0) \\
\hline & $1018(19)$ & $987(22)$ & & $1271(0)$ & $1221(0)$ \\
\hline & 1027(14) & 986(4) & & $1273(0)$ & $1222(0)$ \\
\hline & $1065(0)$ & 1037(4) & & $1388(0)$ & 1344(0) \\
\hline & $1070(0)$ & 1039(0) & & 1393(0) & $1354(0)$ \\
\hline & 1134(1) & 1102(9) & & $1446(2)$ & 1402(3) \\
\hline & 1135(3) & 1104(1) & & 1455(1) & $1403(0)$ \\
\hline & $1381(2)$ & $1350(2)$ & & 1901(1244) & $1798(963)$ \\
\hline & 1396(3) & $1356(0)$ & & $3233(0)$ & $3165(0)$ \\
\hline & $1456(1)$ & $1400(1)$ & & $3243(0)$ & $3168(0)$ \\
\hline & 1468(1) & 1401(4) & & $3248(0)$ & $3180(0)$ \\
\hline & $1918(100)$ & 1820(89) & & $3254(0)$ & 3181(0) \\
\hline & $3229(0)$ & $3163(1)$ & & & \\
\hline & $3238(0)$ & $3167(0)$ & & & \\
\hline & $3245(1)$ & $3178(0)$ & & & \\
\hline & $3254(0)$ & $3178(0)$ & & & \\
\hline & $3259(1)$ & 3190(0) & & & \\
\hline & $3266(0)$ & $3190(0)$ & & & \\
\hline
\end{tabular}


Table S10. Harmonic vibrational frequencies (in $\mathrm{cm}^{-1}$ ) and infrared intensities (in parentheses in $\mathrm{km} / \mathrm{mol}$ ) for singlet $C_{2 h}$ trans-unbridged $\mathrm{Cp}_{2} \mathrm{Fe}_{2}(\mathrm{CO})_{2}$ (IIIc)

\begin{tabular}{|c|c|c|c|c|c|}
\hline & B3LYP & BP86 & & B3LYP & BP86 \\
\hline \multirow[t]{21}{*}{$a_{g}$} & $57(0)$ & $56(0)$ & \multirow[t]{15}{*}{$b_{g}$} & $66 \mathbf{i}$ & $73 i$ \\
\hline & $131(0)$ & $119(0)$ & & $136(0)$ & $134(0)$ \\
\hline & $198(0)$ & 191(0) & & $332(0)$ & $350(0)$ \\
\hline & $357(0)$ & $355(0)$ & & $510(0)$ & $515(0)$ \\
\hline & $404(0)$ & $415(0)$ & & $585(0)$ & $550(0)$ \\
\hline & $531(0)$ & $549(0)$ & & $813(0)$ & $769(0)$ \\
\hline & $572(0)$ & $566(0)$ & & $836(0)$ & 799(0) \\
\hline & $595(0)$ & $582(0)$ & & $907(0)$ & $863(0)$ \\
\hline & $824(0)$ & $752(0)$ & & $1007(0)$ & $958(0)$ \\
\hline & $835(0)$ & $796(0)$ & & $1062(0)$ & $1023(0)$ \\
\hline & $856(0)$ & $820(0)$ & & $1264(0)$ & $1205(0)$ \\
\hline & $916(0)$ & $868(0)$ & & $1372(0)$ & $1308(0)$ \\
\hline & $1004(0)$ & $969(0)$ & & $1460(0)$ & $1403(0)$ \\
\hline & $1069(0)$ & 1037(0) & & $3240(0)$ & $3162(0)$ \\
\hline & $1124(0)$ & 1084(0) & & $3256(0)$ & $3177(0)$ \\
\hline & $1400(0)$ & 1358(0) & \multirow[t]{22}{*}{$\mathrm{b}_{\mathrm{u}}$} & $99(0)$ & $88(0)$ \\
\hline & $1433(0)$ & 1372(0) & & $166(4)$ & $173(7)$ \\
\hline & 2013(0) & 1920(0) & & $351(3)$ & $347(6)$ \\
\hline & $3246(0)$ & $3167(0)$ & & $388(5)$ & $397(2)$ \\
\hline & $3261(0)$ & $3177(0)$ & & $526(113)$ & $517(86)$ \\
\hline & $3289(0)$ & $3186(0)$ & & $535(37)$ & $557(32)$ \\
\hline \multirow[t]{16}{*}{$a_{u}$} & $84 i$ & $\mathbf{7 8 i}$ & & 592(16) & $576(27)$ \\
\hline & $40 i$ & $39 \mathbf{i}$ & & $823(80)$ & $767(10)$ \\
\hline & $94(0)$ & $91(0)$ & & $826(16)$ & $783(40)$ \\
\hline & 287(9) & $302(7)$ & & $855(32)$ & $821(42)$ \\
\hline & $440(11)$ & $478(17)$ & & $918(11)$ & $866(13)$ \\
\hline & $592(0)$ & $559(1)$ & & $1004(13)$ & $970(10)$ \\
\hline & $822(0)$ & $781(0)$ & & $1068(0)$ & $1035(2)$ \\
\hline & $838(2)$ & $802(3)$ & & 1122(11) & 1082(12) \\
\hline & $907(2)$ & $863(3)$ & & 1399(19) & 1357(6) \\
\hline & $1006(21)$ & $959(23)$ & & 1434(1) & 1372(2) \\
\hline & 1063(4) & $1024(7)$ & & $1988(2620)$ & 1904(2035) \\
\hline & $1264(0)$ & $1206(0)$ & & $3246(3)$ & $3166(7)$ \\
\hline & $1374(0)$ & $1312(0)$ & & $3261(2)$ & $3175(7)$ \\
\hline & $1460(3)$ & $1402(2)$ & & $3287(3)$ & $3185(2)$ \\
\hline & $3240(1)$ & $3162(0)$ & & & \\
\hline & $3255(0)$ & $3177(1)$ & & & \\
\hline
\end{tabular}


Table S11. Harmonic vibrational frequencies (in $\mathrm{cm}^{-1}$ ) and infrared intensities (in parentheses in $\mathrm{km} / \mathrm{mol}$ ) for triplet $C_{i}$ trans-unbridged $\mathrm{Cp}_{2} \mathrm{Fe}_{2}(\mathrm{CO})_{2}$ (IIId)

\begin{tabular}{|c|c|c|c|c|c|}
\hline & B3LYP & BP86 & & B3LY & BP86 \\
\hline \multirow{36}{*}{$\mathrm{a}_{\mathrm{g}}$} & $59(0)$ & $74(0)$ & $a_{u}$ & 33i & $65 i$ \\
\hline & $88(0)$ & $98(0)$ & & $55(0)$ & $3(2)$ \\
\hline & $113(0)$ & $123(0)$ & & $69(1)$ & $78(1)$ \\
\hline & $113(0)$ & $133(0)$ & & $90(1)$ & $100(0)$ \\
\hline & $147(0)$ & $180(0)$ & & $141(0)$ & $172(2)$ \\
\hline & $264(0)$ & $296(0)$ & & 264(1) & $228(29)$ \\
\hline & $286(0)$ & $324(0)$ & & $286(7)$ & $329(14)$ \\
\hline & $317(0)$ & $387(0)$ & & $316(4)$ & $349(3)$ \\
\hline & $431(0)$ & $462(0)$ & & $395(1)$ & $408(4)$ \\
\hline & $516(0)$ & $530(0)$ & & $514(75)$ & $507(9)$ \\
\hline & $540(0)$ & $544(0)$ & & $525(36)$ & $539(80)$ \\
\hline & $578(0)$ & $547(0)$ & & $571(1)$ & $545(37)$ \\
\hline & $588(0)$ & $563(0)$ & & $587(3)$ & $563(3)$ \\
\hline & $744(0)$ & $665(0)$ & & $760(61)$ & $679(26)$ \\
\hline & 791(0) & $759(0)$ & & 787(126) & 751(51) \\
\hline & $813(0)$ & $785(0)$ & & $813(23)$ & $786(14)$ \\
\hline & $832(0)$ & 793(0) & & $835(9)$ & 797(3) \\
\hline & $837(0)$ & $809(0)$ & & $839(1)$ & 811(19) \\
\hline & $880(0)$ & $839(0)$ & & $877(5)$ & $833(3)$ \\
\hline & $914(0)$ & $873(0)$ & & $914(12)$ & $873(13)$ \\
\hline & $999(0)$ & $959(0)$ & & 999(31) & 957(11) \\
\hline & $1023(0)$ & $985(0)$ & & 1022(44) & $985(33)$ \\
\hline & $1062(0)$ & $1022(0)$ & & 1060(9) & 1021(5) \\
\hline & $1069(0)$ & $1036(0)$ & & 1069(1) & $1036(2)$ \\
\hline & $1122(0)$ & 1083(0) & & 1121(6) & 1082(12) \\
\hline & $1264(0)$ & $1206(0)$ & & 1264(1) & $1206(1)$ \\
\hline & $1380(0)$ & $1328(0)$ & & $1377(3)$ & $1329(1)$ \\
\hline & $1386(0)$ & $1342(0)$ & & 1386(4) & $1343(1)$ \\
\hline & $1436(0)$ & 1377(0) & & 1435(4) & 1377(12) \\
\hline & $1451(0)$ & $1390(0)$ & & $1451(7)$ & 1390(5) \\
\hline & $2015(0)$ & 1919(0) & & 1998(2536) & 1897(2330) \\
\hline & $3162(0)$ & 3033(0) & & $3160(4)$ & 3029(3) \\
\hline & $3237(0)$ & 3161(0) & & $3237(1)$ & 3161(2) \\
\hline & $3246(0)$ & $3170(0)$ & & $3247(1)$ & $3170(2)$ \\
\hline & $3254(0)$ & $3175(0)$ & & $3254(2)$ & $3175(4)$ \\
\hline & $3262(0)$ & $3185(0)$ & & $3262(1)$ & $3185(0)$ \\
\hline
\end{tabular}


Table S12. Harmonic vibrational frequencies (in $\mathrm{cm}^{-1}$ ) and infrared intensities (in parentheses in $\mathrm{km} / \mathrm{mol}$ ) for singlet $C_{2 v}$ cis-unbridged $\mathrm{Cp}_{2} \mathrm{Fe}_{2}(\mathrm{CO})_{2}$ (IIIe)

\begin{tabular}{|c|c|c|c|}
\hline & B3LYP & & BP86 \\
\hline \multirow[t]{21}{*}{$a_{1}$} & 111(0) & $\mathrm{b}_{1}$ & 138i \\
\hline & $130(0)$ & & $36 \mathbf{i}$ \\
\hline & $164(0)$ & & $311(16)$ \\
\hline & $324(1)$ & & $365(9)$ \\
\hline & $384(3)$ & & $584(5)$ \\
\hline & $521(28)$ & & $800(13)$ \\
\hline & $557(1)$ & & $834(8)$ \\
\hline & $590(2)$ & & $894(1)$ \\
\hline & $820(47)$ & & 1023(37) \\
\hline & $837(1)$ & & 1066(6) \\
\hline & $850(8)$ & & $1269(0)$ \\
\hline & $916(6)$ & & 1391(1) \\
\hline & $1001(18)$ & & $1457(0)$ \\
\hline & 1064(1) & & $3233(5)$ \\
\hline & $1129(8)$ & & $3248(0)$ \\
\hline & $1383(0)$ & $b_{2}$ & $50(1)$ \\
\hline & $1447(0)$ & & 198(4) \\
\hline & $2047(1508)$ & & $370(1)$ \\
\hline & $3235(0)$ & & 461(16) \\
\hline & $3257(0)$ & & $516(45)$ \\
\hline & $3285(1)$ & & $556(46)$ \\
\hline \multirow[t]{16}{*}{$\mathrm{a}_{2}$} & $55 \mathbf{i}$ & & 723(79) \\
\hline & $7(0)$ & & $815(42)$ \\
\hline & $146(0)$ & & $838(9)$ \\
\hline & $334(0)$ & & $880(32)$ \\
\hline & $495(0)$ & & $995(7)$ \\
\hline & $590(0)$ & & 1054(105) \\
\hline & $811(0)$ & & $1067(30)$ \\
\hline & $834(0)$ & & $1352(715)$ \\
\hline & $893(0)$ & & 1391(102) \\
\hline & 1018(0) & & $1566(2051)$ \\
\hline & 1059(0) & & 2443(4137) \\
\hline & $1267(0)$ & & $3234(2)$ \\
\hline & 1392(0) & & $3259(54)$ \\
\hline & $1456(0)$ & & $3283(270)$ \\
\hline & $3233(0)$ & & \\
\hline & $3248(0)$ & & \\
\hline
\end{tabular}


Table S13. Harmonic vibrational frequencies (in $\mathrm{cm}^{-1}$ ) and infrared intensities (in parentheses in $\mathrm{km} / \mathrm{mol}$ ) for triplet $C_{2 v}$ cis-unbridged $\mathrm{Cp}_{2} \mathrm{Fe}_{2}(\mathrm{CO})_{2}$ (IIIf)

\begin{tabular}{|c|c|c|c|c|c|}
\hline & B3LYP & BP86 & & B3LYP & BP86 \\
\hline \multirow[t]{21}{*}{$a_{1}$} & $100(0)$ & $90(0)$ & \multirow[t]{15}{*}{$b_{1}$} & $24 \mathrm{i}$ & 247i \\
\hline & $115(0)$ & $117(0)$ & & $72(0)$ & $44(8)$ \\
\hline & $142(0)$ & $157(1)$ & & $301(4)$ & $79(0)$ \\
\hline & $326(0)$ & $343(8)$ & & $428(5)$ & $426(1)$ \\
\hline & $356(2)$ & $372(0)$ & & $580(0)$ & $528(36)$ \\
\hline & $518(30)$ & $542(15)$ & & $819(1)$ & $730(122)$ \\
\hline & $539(1)$ & $552(5)$ & & $838(1)$ & 796(1) \\
\hline & $593(1)$ & $564(4)$ & & $901(0)$ & $841(27)$ \\
\hline & $816(49)$ & 793(23) & & 1023(21) & $932(122)$ \\
\hline & 833(9) & $798(5)$ & & 1069(2) & $1015(0)$ \\
\hline & $845(18)$ & 812(17) & & $1272(0)$ & 1219(3) \\
\hline & 911(3) & $872(6)$ & & 1390(1) & $1230(109)$ \\
\hline & 1014(17) & 983(15) & & 1458(3) & 1398(6) \\
\hline & 1069(0) & 1037(0) & & $3233(1)$ & $3154(0)$ \\
\hline & 1133(4) & $1100(8)$ & & $3248(2)$ & 3172(0) \\
\hline & 1394(1) & 1355(1) & \multirow[t]{22}{*}{$b_{2}$} & 132i & $127 i$ \\
\hline & $1450(0)$ & 1397(0) & & $89(2)$ & $117(0)$ \\
\hline & 2049(1589) & 1951(1289) & & $316(6)$ & $334(4)$ \\
\hline & $3234(0)$ & $3154(0)$ & & $324(43)$ & $438(3)$ \\
\hline & $3258(0)$ & 3183(0) & & $507(86)$ & $520(43)$ \\
\hline & 3279(2) & 3201(4) & & $532(20)$ & 544(27) \\
\hline \multirow[t]{16}{*}{$\mathrm{a}_{2}$} & $49 \mathrm{i}$ & $92 \mathrm{i}$ & & $586(10)$ & 593(4) \\
\hline & $41 i$ & $48 i$ & & $783(47)$ & $745(1)$ \\
\hline & $110(0)$ & $130(0)$ & & $814(76)$ & 791(36) \\
\hline & $290(0)$ & $295(0)$ & & $834(4)$ & 798(28) \\
\hline & $428(0)$ & $496(0)$ & & $890(15)$ & $853(1)$ \\
\hline & $569(0)$ & $544(0)$ & & 1011(8) & $980(6)$ \\
\hline & $817(0)$ & 787(0) & & 1068(1) & $1035(5)$ \\
\hline & $832(0)$ & $804(0)$ & & $1135(5)$ & $1105(31)$ \\
\hline & $902(0)$ & $856(0)$ & & 1395(8) & $1359(5)$ \\
\hline & 1017(0) & 981(0) & & $1447(0)$ & 1397(14) \\
\hline & $1058(0)$ & $1025(0)$ & & $1976(404)$ & 1905(367) \\
\hline & $1269(0)$ & $1217(0)$ & & $3233(3)$ & $3154(3)$ \\
\hline & 1384(0) & $1349(0)$ & & $3257(1)$ & 3183(1) \\
\hline & $1456(0)$ & 1396(0) & & $3272(0)$ & $3200(0)$ \\
\hline & $3233(0)$ & $3154(0)$ & & & \\
\hline & $3248(0)$ & $3172(0)$ & & & \\
\hline
\end{tabular}


Table S14. Harmonic vibrational frequencies (in $\mathrm{cm}^{-1}$ ) and infrared intensities (in parentheses in $\mathrm{km} / \mathrm{mol}$ ) for triplet $C_{s}$ monobridged $\mathrm{Cp}_{2} \mathrm{Fe}_{2}(\mathrm{CO})(\mu-\mathrm{CO})($ IIIg)

\begin{tabular}{|c|c|c|c|c|c|}
\hline & B3LYP & BP86 & & B3LYP & BP86 \\
\hline \multirow[t]{41}{*}{$a^{\prime}$} & $80(0)$ & $89(0)$ & a" & 334i & $92 \mathrm{i}$ \\
\hline & $100(1)$ & $107(0)$ & & $26 \mathbf{i}$ & $13(0)$ \\
\hline & $117(1)$ & $121(2)$ & & $16 \mathbf{i}$ & $27(0)$ \\
\hline & $163(0)$ & $176(0)$ & & $40(0)$ & $42(0)$ \\
\hline & 203(4) & $223(0)$ & & 70(1) & 92(0) \\
\hline & $248(1)$ & $272(2)$ & & $220(0)$ & $258(3)$ \\
\hline & $325(30)$ & $369(3)$ & & $257(5)$ & $335(4)$ \\
\hline & $345(0)$ & $345(4)$ & & $311(5)$ & $378(0)$ \\
\hline & $368(2)$ & 395(2) & & $413(0)$ & $456(2)$ \\
\hline & 421(38) & $459(21)$ & & $580(0)$ & $549(0)$ \\
\hline & 494(50) & $514(8)$ & & $601(0)$ & $562(1)$ \\
\hline & $534(88)$ & $545(42)$ & & $785(10)$ & $772(0)$ \\
\hline & 591(21) & $558(1)$ & & $795(0)$ & $770(2)$ \\
\hline & 593(1) & $567(7)$ & & $834(2)$ & $801(0)$ \\
\hline & $603(0)$ & $597(19)$ & & $842(0)$ & $804(1)$ \\
\hline & 784(22) & 738(3) & & $887(1)$ & $844(1)$ \\
\hline & 796(130) & 772(55) & & $904(4)$ & $862(2)$ \\
\hline & $815(51)$ & $765(25)$ & & 1009(18) & 971(5) \\
\hline & $830(12)$ & 791(24) & & 1016(29) & $979(27)$ \\
\hline & $843(3)$ & $808(23)$ & & 1063(2) & $1027(5)$ \\
\hline & $848(0)$ & $812(3)$ & & $1065(3)$ & 1031(1) \\
\hline & $887(0)$ & $849(5)$ & & $1266(0)$ & $1213(0)$ \\
\hline & $899(6)$ & $855(1)$ & & $1270(0)$ & $1219(0)$ \\
\hline & $997(8)$ & $969(5)$ & & 1369(13) & $1324(0)$ \\
\hline & 1019(16) & $990(12)$ & & 1386(1) & $1340(2)$ \\
\hline & $1066(0)$ & 1033(2) & & 1453(1) & $1397(1)$ \\
\hline & 1067(0) & 1037(1) & & 1470(0) & $1408(1)$ \\
\hline & $1125(3)$ & 1089(9) & & $3228(1)$ & $3157(1)$ \\
\hline & $1133(0)$ & $1100(6)$ & & $3239(4)$ & $3165(0)$ \\
\hline & 1381(1) & $1337(5)$ & & $3244(1)$ & $3174(0)$ \\
\hline & 1398(2) & 1362(1) & & $3253(1)$ & $3178(0)$ \\
\hline & $1435(2)$ & 1379(5) & & & \\
\hline & $1455(1)$ & $1405(1)$ & & & \\
\hline & 1906(366) & 1795(344) & & & \\
\hline & $2040(1440)$ & 1959(1164) & & & \\
\hline & $3201(1)$ & $3131(0)$ & & & \\
\hline & $3221(0)$ & $3149(0)$ & & & \\
\hline & $3240(1)$ & $3168(1)$ & & & \\
\hline & $3245(0)$ & 3171(1) & & & \\
\hline & $3257(1)$ & $3186(1)$ & & & \\
\hline & $3262(0)$ & $3186(1)$ & & & \\
\hline
\end{tabular}


Table S15. Harmonic vibrational frequencies (in $\mathrm{cm}^{-1}$ ) and infrared intensities (in parentheses in $\mathrm{km} / \mathrm{mol}$ ) for triplet $C_{1}$ bridged $\mathrm{Cp}_{2} \mathrm{Fe}_{2}(\mu-\mathrm{CO})(\mathbf{I V a})$

\begin{tabular}{ccccc}
\hline B3LYP & BP86 & B3LYP & BP86 \\
asi(1) & $8(0)$ & a & $895(1)$ & $851(3)$ \\
$13(0)$ & $35(0)$ & $900(2)$ & $853(1)$ \\
$28(0)$ & $57(0)$ & $1013(20)$ & $979(19)$ \\
$39(0)$ & $60(0)$ & $1016(14)$ & $983(11)$ \\
$50(1)$ & $91(0)$ & $1017(14)$ & $986(13)$ \\
$93(1)$ & $107(1)$ & $1019(14)$ & $987(13)$ \\
$168(2)$ & $180(3)$ & $1063(0)$ & $1031(0)$ \\
$173(0)$ & $191(2)$ & $1065(0)$ & $1033(0)$ \\
$264(4)$ & $269(1)$ & $1070(0)$ & $1040(0)$ \\
$282(5)$ & $290(2)$ & $1071(1)$ & $1040(1)$ \\
$296(1)$ & $319(2)$ & $1131(2)$ & $1098(8)$ \\
$305(3)$ & $336(3)$ & $1133(6)$ & $1101(5)$ \\
$361(17)$ & $360(1)$ & $1270(0)$ & $1220(0)$ \\
$406(9)$ & $377(6)$ & $1273(0)$ & $1220(0)$ \\
$431(3)$ & $429(2)$ & $1378(0)$ & $1333(0)$ \\
$444(12)$ & $465(9)$ & $1379(1)$ & $1341(0)$ \\
$536(5)$ & $541(6)$ & $1385(1)$ & $1347(1)$ \\
$578(1)$ & $550(1)$ & $1387(0)$ & $1356(1)$ \\
$592(2)$ & $551(1)$ & $1449(1)$ & $1398(3)$ \\
$595(1)$ & $567(1)$ & $1456(2)$ & $1399(2)$ \\
$610(1)$ & $574(2)$ & $1459(2)$ & $1404(1)$ \\
$792(91)$ & $757(54)$ & $1461(1)$ & $1413(2)$ \\
$796(49)$ & $764(29)$ & $1949(830)$ & $1864(638)$ \\
$798(11)$ & $765(0)$ & $3229(0)$ & $3157(0)$ \\
$800(12)$ & $770(15)$ & $3229(0)$ & $3160(0)$ \\
$803(19)$ & $772(21)$ & $3232(0)$ & $3161(0)$ \\
$811(6)$ & $777(7)$ & $3234(0)$ & $3164(0)$ \\
$834(3)$ & $800(2)$ & $3244(1)$ & $3172(1)$ \\
$837(1)$ & $801(1)$ & $3245(0)$ & $3174(1)$ \\
$842(0)$ & $809(0)$ & $3246(1)$ & $3174(1)$ \\
$845(1)$ & $810(0)$ & $3249(1)$ & $3177(1)$ \\
$879(1)$ & $832(1)$ & $3258(1)$ & $3185(1)$ \\
$879(1)$ & $838(0)$ & $3259(0)$ & $3187(0)$ \\
\hline & & & \\
\hline
\end{tabular}


Table S16. Harmonic vibrational frequencies (in $\mathrm{cm}^{-1}$ ) and infrared intensities (in parentheses in $\mathrm{km} / \mathrm{mol}$ ) for singlet $C_{\mathrm{s}}$ bridged $\mathrm{Cp}_{2} \mathrm{Fe}_{2}(\mu-\mathrm{CO})(\mathbf{I V b})$

\begin{tabular}{|c|c|c|c|c|c|}
\hline & B3LYP & BP86 & & B3LYP & BP86 \\
\hline \multirow{34}{*}{$a_{-}$} & $5 \mathbf{i}(0)$ & 32i $(0)$ & a" & $28(0)$ & $44 i(19)$ \\
\hline & $50(2)$ & $84(0)$ & & $98(1)$ & $46(0)$ \\
\hline & $74(1)$ & $69(1)$ & & $122(0)$ & $128(0)$ \\
\hline & $186(1)$ & 193(0) & & $214(0)$ & $213(5)$ \\
\hline & $361(4)$ & $363(10)$ & & $353(8)$ & $364(14)$ \\
\hline & $370(21)$ & $367(23)$ & & $385(0)$ & 407(1) \\
\hline & $452(11)$ & 445(9) & & $404(0)$ & $421(2)$ \\
\hline & $478(1)$ & $465(4)$ & & $473(34)$ & $477(1)$ \\
\hline & $527(8)$ & $535(2)$ & & 574(1) & $536(10)$ \\
\hline & $584(2)$ & $551(3)$ & & $583(0)$ & $550(0)$ \\
\hline & $586(2)$ & $557(2)$ & & 792(0) & $756(0)$ \\
\hline & $789(4)$ & $749(6)$ & & $808(49)$ & $773(45)$ \\
\hline & $815(5)$ & $778(3)$ & & 831(11) & 788(3) \\
\hline & $834(4)$ & 797(1) & & $832(48)$ & $793(62)$ \\
\hline & $836(2)$ & 801(4) & & $835(0)$ & $800(18)$ \\
\hline & $847(3)$ & $811(4)$ & & $880(9)$ & $834(18)$ \\
\hline & $890(3)$ & $845(2)$ & & $891(0)$ & $846(0)$ \\
\hline & $894(0)$ & $847(0)$ & & $994(8)$ & $955(16)$ \\
\hline & $998(23)$ & $960(18)$ & & $1019(0)$ & $987(0)$ \\
\hline & 1019(39) & 987(38) & & 1054(0) & $1024(0)$ \\
\hline & $1059(2)$ & $1025(2)$ & & $1057(0)$ & $1026(0)$ \\
\hline & $1060(1)$ & $1030(0)$ & & $1115(16)$ & $1076(5)$ \\
\hline & $1122(5)$ & 1085(3) & & $1265(0)$ & 1214(0) \\
\hline & $1265(0)$ & $1214(0)$ & & $1357(30)$ & $1309(74)$ \\
\hline & 1373(3) & 1323(2) & & 1379(0) & $1345(0)$ \\
\hline & $1388(0)$ & 1351(0) & & $1428(5)$ & $1377(1)$ \\
\hline & $1429(1)$ & $1377(2)$ & & $1449(0)$ & $1395(0)$ \\
\hline & $1450(0)$ & $1395(0)$ & & $3217(0)$ & $3141(0)$ \\
\hline & 1894(635) & 1789(474) & & $3239(0)$ & $3155(0)$ \\
\hline & $3217(0)$ & $3141(0)$ & & $3244(2)$ & 3161(1) \\
\hline & $3238(0)$ & $3154(0)$ & & $3251(0)$ & $3167(0)$ \\
\hline & $3244(1)$ & $3161(2)$ & & $3260(1)$ & $3176(7)$ \\
\hline & $3251(0)$ & $3167(2)$ & & & \\
\hline & $3261(0)$ & $3176(0)$ & & & \\
\hline
\end{tabular}


Table S17 Harmonic vibrational frequencies (in $\mathrm{cm}^{-1}$ ) and infrared intensities (in parentheses in $\mathrm{km} / \mathrm{mol}$ ) for singlet $C_{1}$ unbridged $\mathrm{Cp}_{2} \mathrm{Fe}_{2}(\mathrm{CO})($ IVc)

\begin{tabular}{|c|c|c|c|c|c|}
\hline & B3LYP & BP86 & & B3LYP & BP86 \\
\hline \multirow[t]{33}{*}{$\mathrm{a}$} & $20(0)$ & $57(0)$ & $\mathrm{a}$ & $887(1)$ & $865(5)$ \\
\hline & $61(1)$ & $75(0)$ & & $908(5)$ & $877(8)$ \\
\hline & $66(0)$ & $92(2)$ & & $951(13)$ & $923(7)$ \\
\hline & $86(0)$ & $101(0)$ & & $969(18)$ & $930(18)$ \\
\hline & $95(0)$ & $147(0)$ & & $991(16)$ & $951(8)$ \\
\hline & $142(1)$ & $184(5)$ & & $996(11)$ & $993(8)$ \\
\hline & $178(2)$ & 202(3) & & $1036(15)$ & $1007(10)$ \\
\hline & $226(1)$ & $242(1)$ & & $1059(4)$ & $1022(2)$ \\
\hline & $316(4)$ & $318(1)$ & & $1059(0)$ & $1025(2)$ \\
\hline & $326(2)$ & $327(9)$ & & $1062(2)$ & $1043(13)$ \\
\hline & $336(9)$ & $379(20)$ & & $1080(12)$ & $1064(13)$ \\
\hline & $380(4)$ & $398(12)$ & & $1115(14)$ & $1080(17)$ \\
\hline & $395(5)$ & $428(4)$ & & $1251(0)$ & $1188(1)$ \\
\hline & $415(2)$ & $456(8)$ & & $1258(0)$ & 1194(0) \\
\hline & $439(5)$ & $488(22)$ & & $1316(21)$ & $1269(5)$ \\
\hline & $537(18)$ & $516(14)$ & & $1355(1)$ & $1288(1)$ \\
\hline & $545(19)$ & $544(16)$ & & $1365(1)$ & $1316(0)$ \\
\hline & $579(6)$ & $547(3)$ & & $1389(7)$ & $1327(1)$ \\
\hline & $581(3)$ & $567(3)$ & & $1410(0)$ & $1349(3)$ \\
\hline & $585(4)$ & $574(1)$ & & $1414(1)$ & $1350(1)$ \\
\hline & $597(24)$ & $607(9)$ & & $1423(2)$ & $1364(0)$ \\
\hline & $667(18)$ & $668(20)$ & & $1455(0)$ & $1389(1)$ \\
\hline & $718(10)$ & $705(1)$ & & $1987(1429)$ & $1908(1084)$ \\
\hline & $786(25)$ & $741(4)$ & & $3130(1)$ & $2627(2)$ \\
\hline & $798(3)$ & $757(3)$ & & $3166(0)$ & 2989(9) \\
\hline & $801(8)$ & $760(13)$ & & $3202(4)$ & $3079(3)$ \\
\hline & 807(19) & $765(1)$ & & $3226(0)$ & $3139(2)$ \\
\hline & $816(15)$ & $777(24)$ & & $3229(0)$ & $3145(1)$ \\
\hline & $823(2)$ & $785(0)$ & & $3234(2)$ & $3149(4)$ \\
\hline & $828(8)$ & $809(6)$ & & $3237(1)$ & $3157(1)$ \\
\hline & $843(20)$ & $818(6)$ & & $3249(1)$ & $3160(5)$ \\
\hline & $850(1)$ & $821(1)$ & & $3249(3)$ & $3168(1)$ \\
\hline & $884(4)$ & $825(1)$ & & $3255(0)$ & $3174(2)$ \\
\hline
\end{tabular}



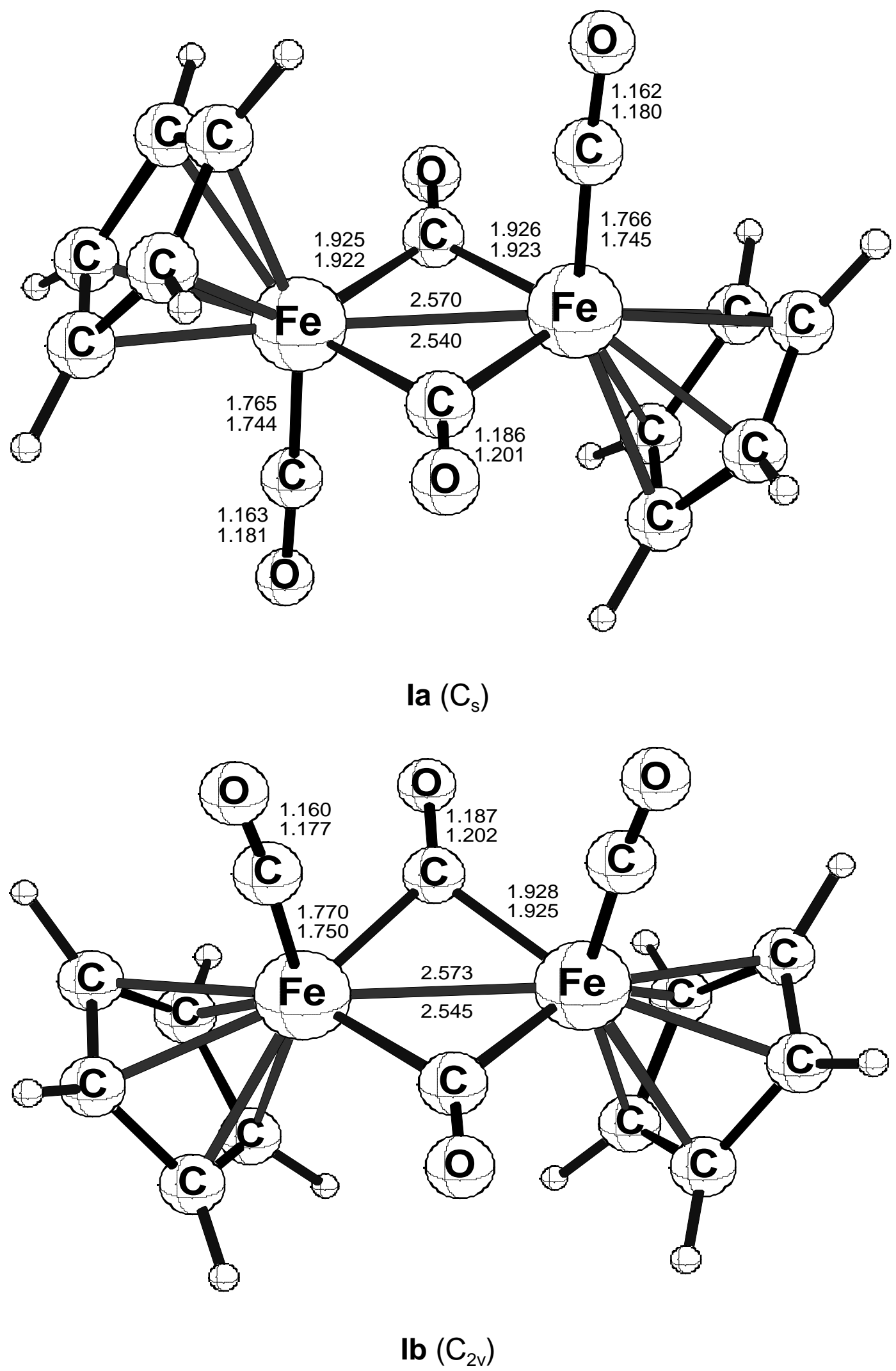

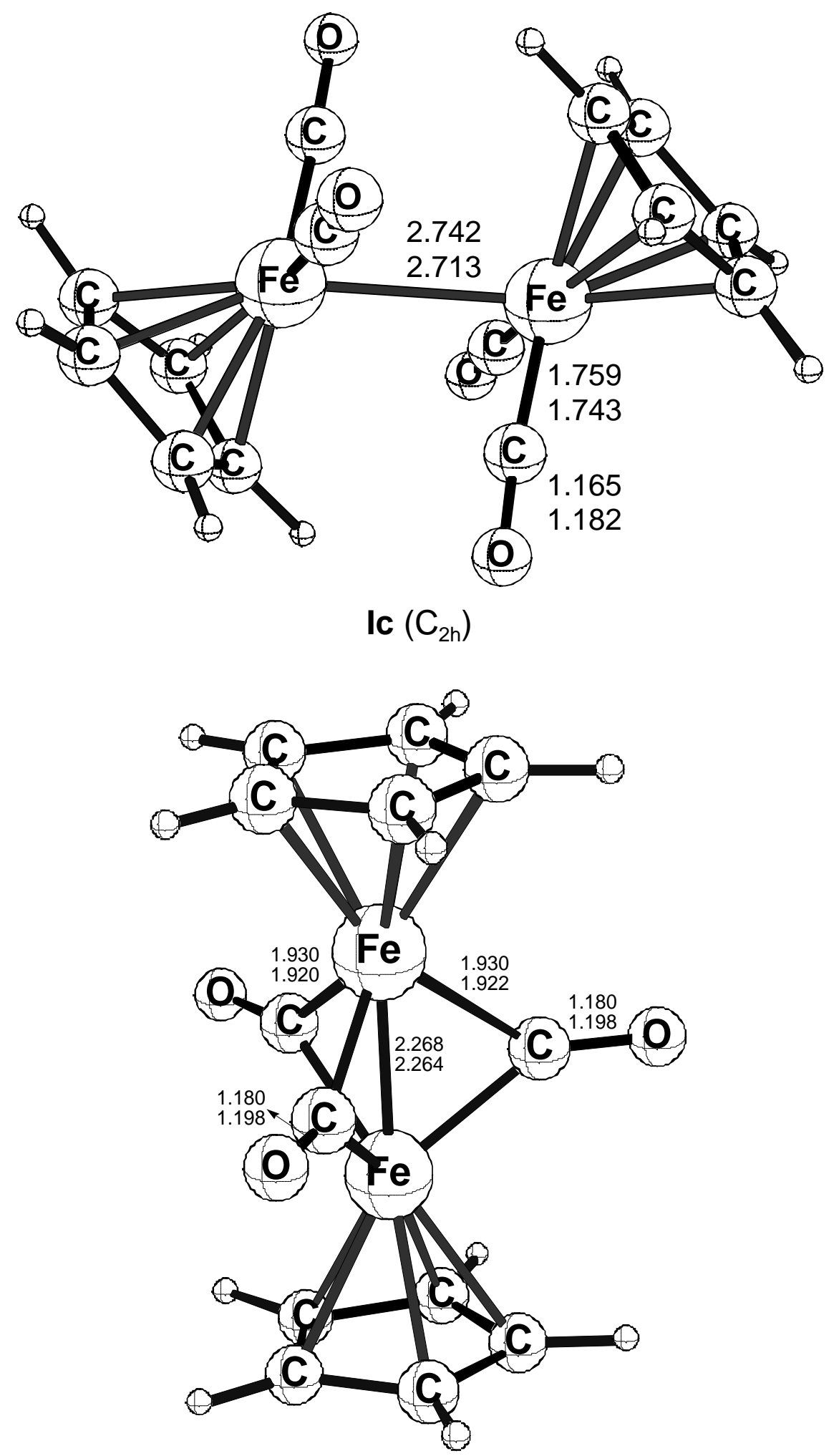

Ila $\left(\mathrm{C}_{2 \mathrm{v}}\right)$ 

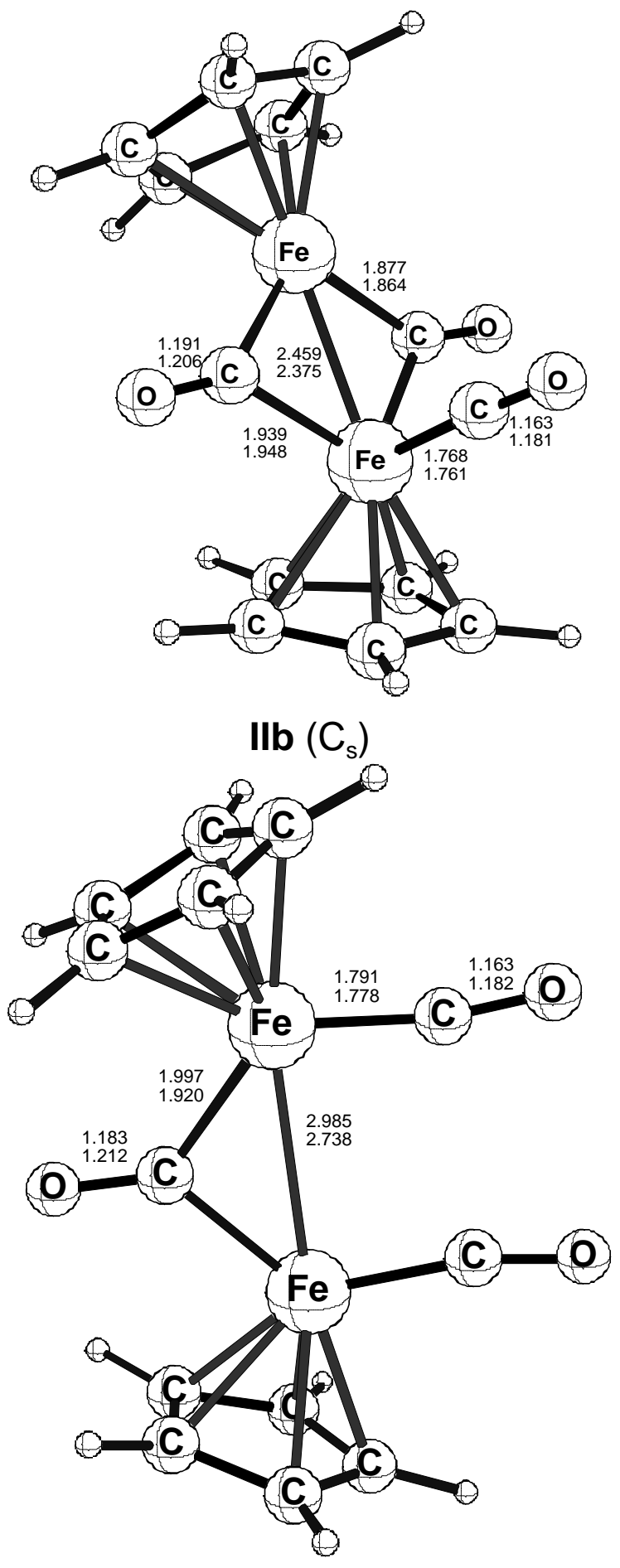

Ilc $\left(\mathrm{C}_{2 \mathrm{v}}\right)$ 

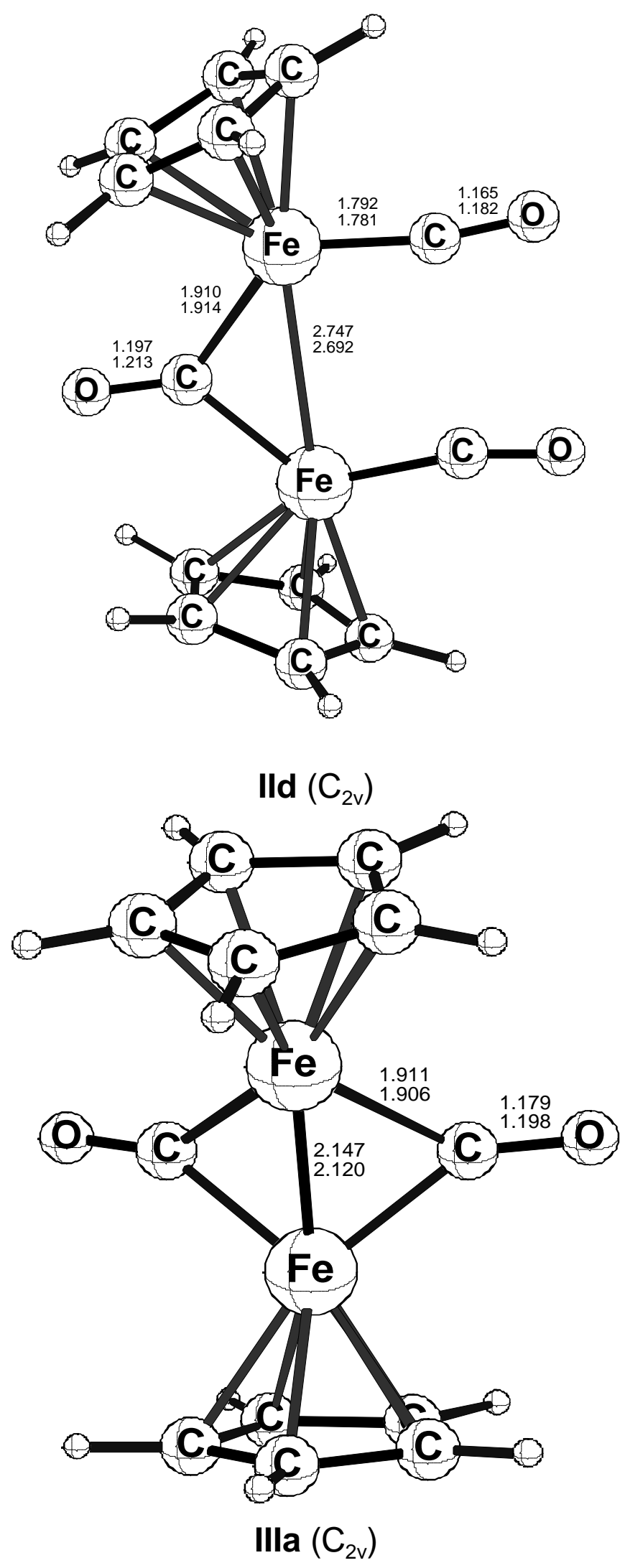


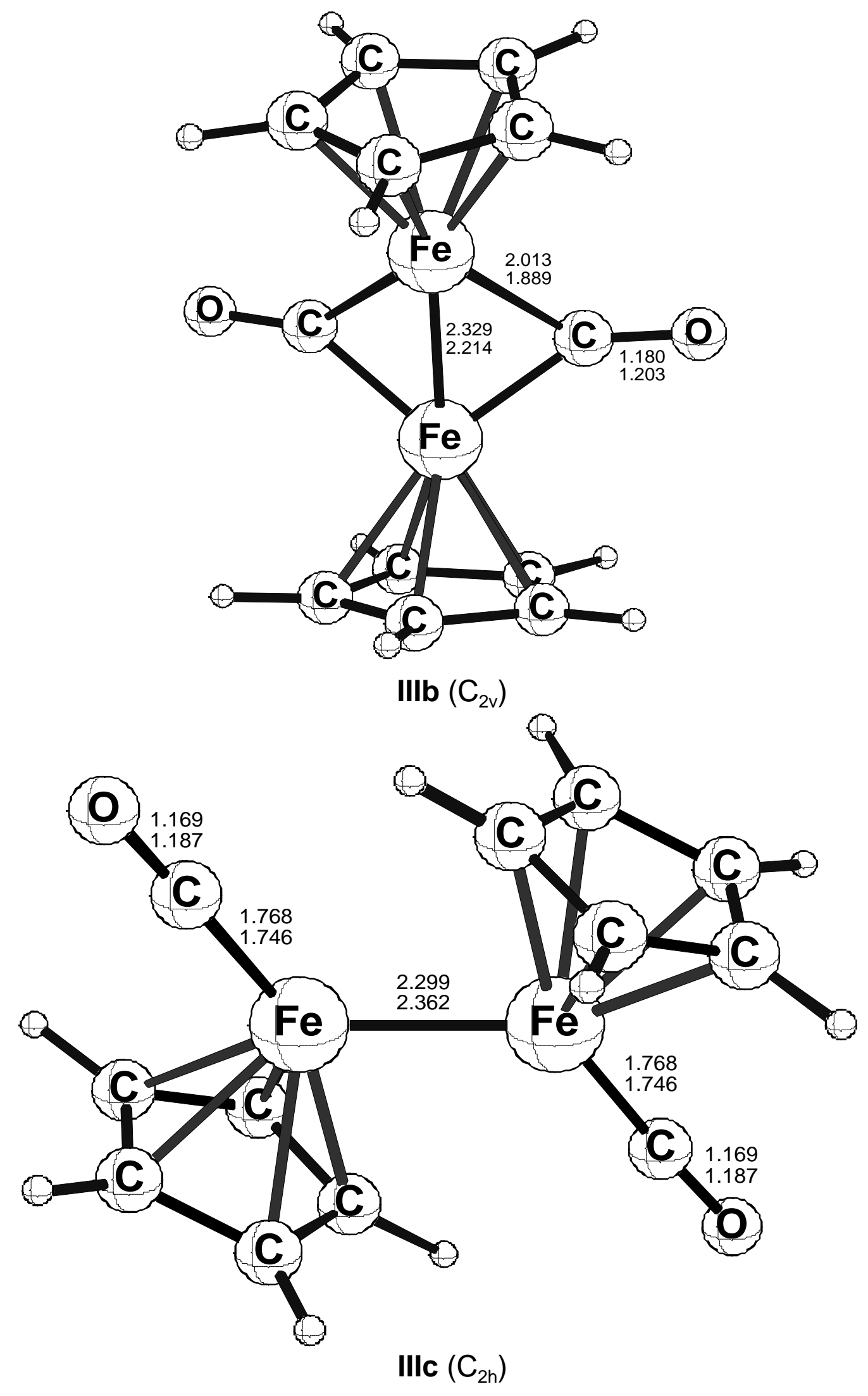



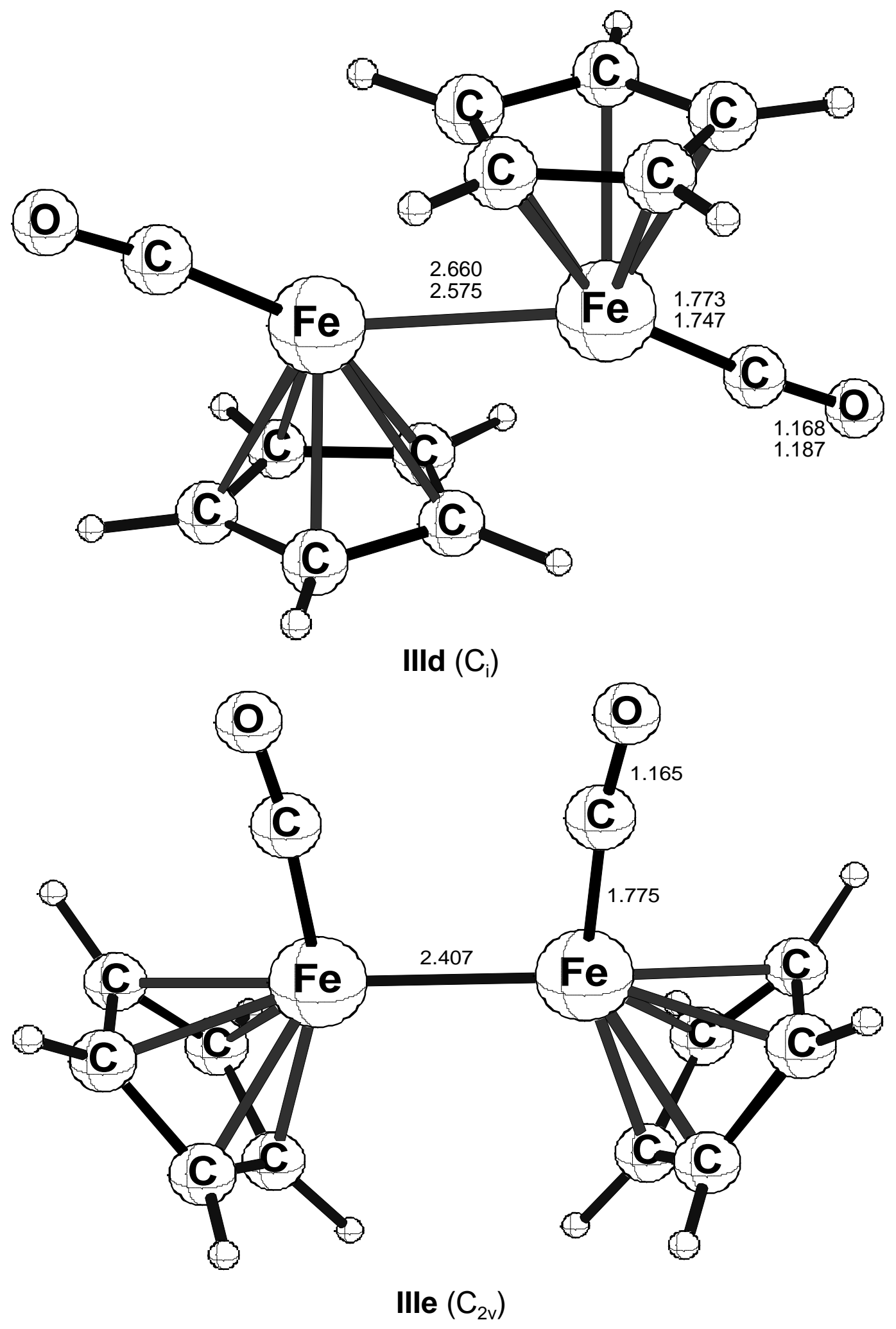

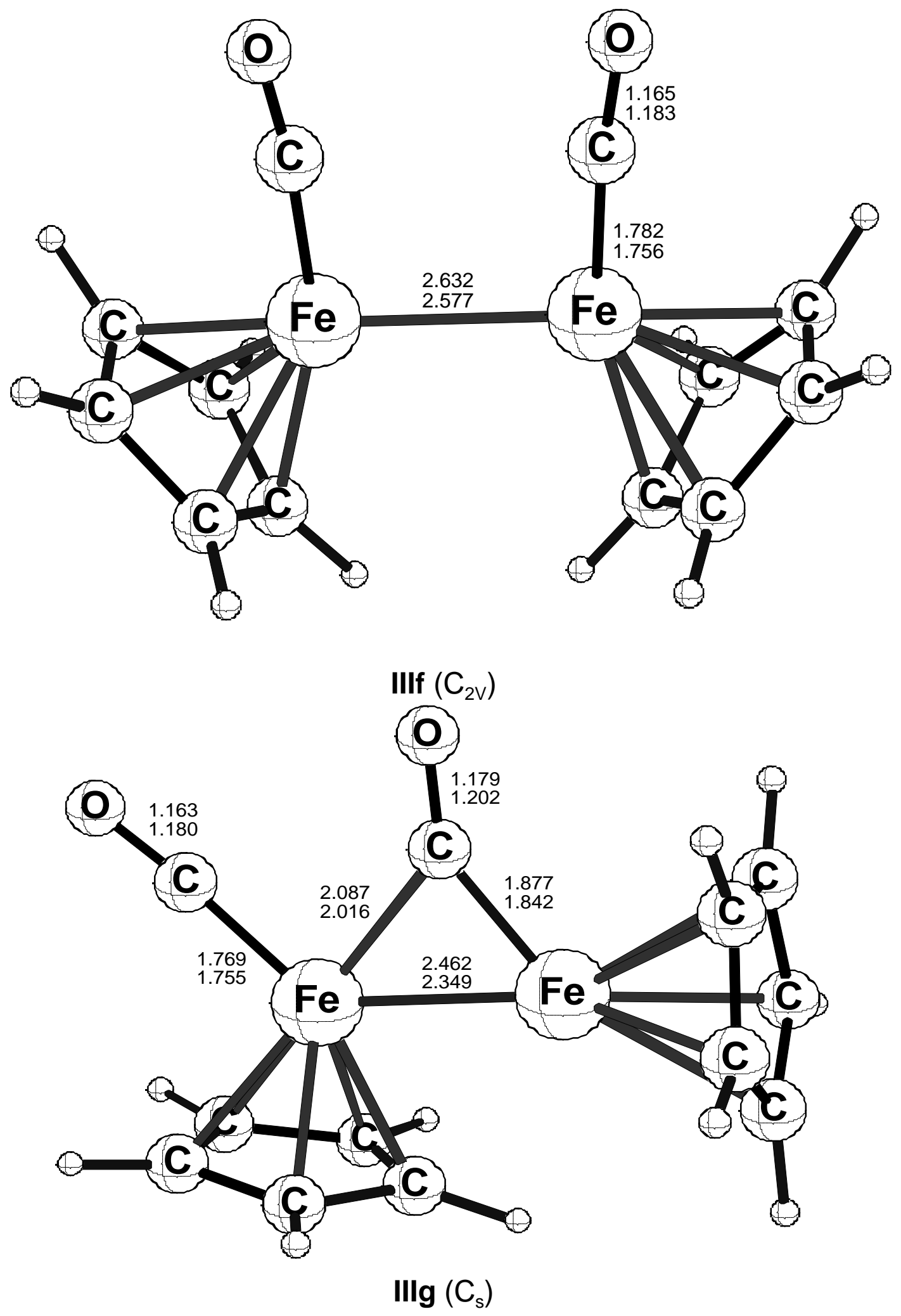

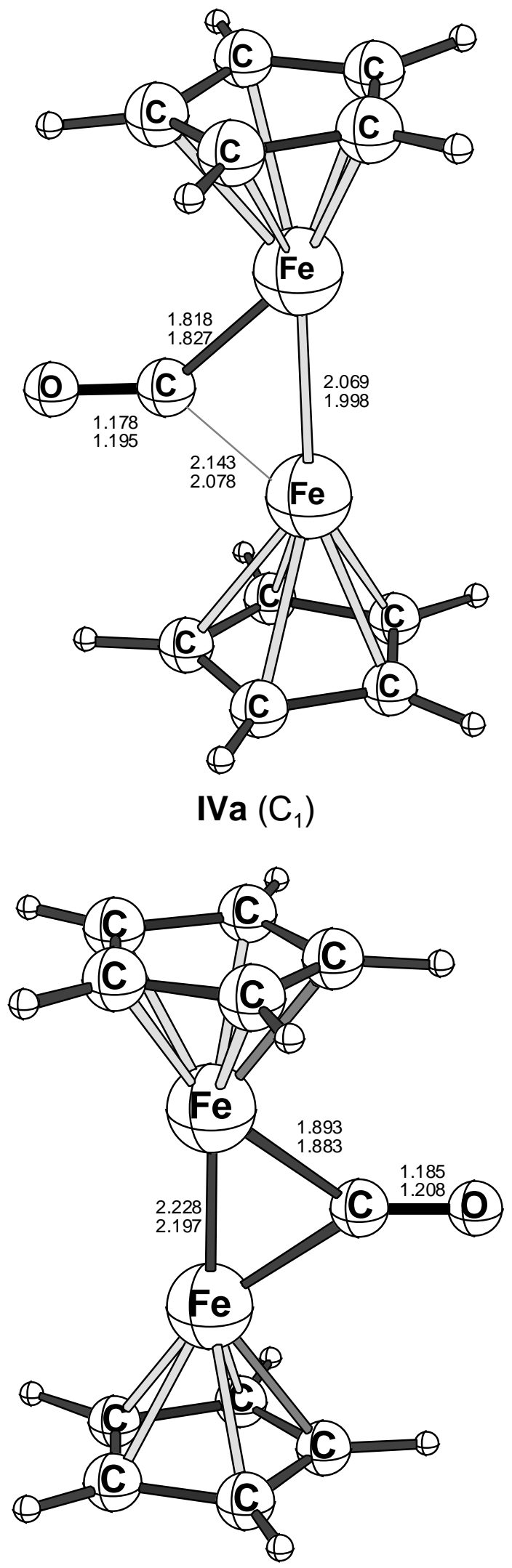

IVb $\left(\mathrm{C}_{\mathrm{s}}\right)$ 


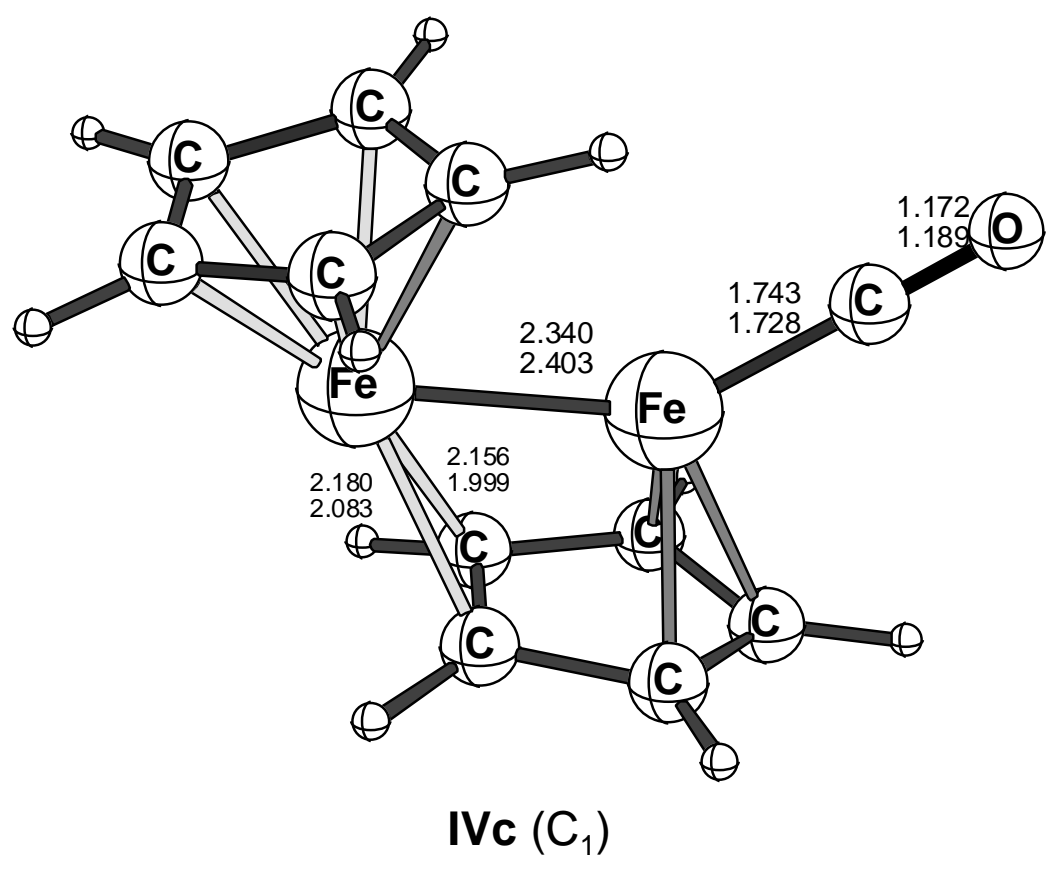

Figure 1. The $17 \mathrm{Cp}_{2} \mathrm{Fe}_{2}(\mathrm{CO})_{n}(n=4,3,2,1)$ structures discussed in this paper. 